This item was submitted to Loughborough's Research Repository by the author.

Items in Figshare are protected by copyright, with all rights reserved, unless otherwise indicated.

\title{
Joint transceiver design for MIMO channel shortening.
}

PLEASE CITE THE PUBLISHED VERSION

PUBLISHER

(C) IEEE

VERSION

VoR (Version of Record)

LICENCE

CC BY-NC-ND 4.0

\section{REPOSITORY RECORD}

Toker, Cenk, Sangarapillai Lambotharan, and Jonathon Chambers. 2019. "Joint Transceiver Design for MIMO Channel Shortening.". figshare. https://hdl.handle.net/2134/5531. 
This item was submitted to Loughborough's Institutional Repository (https://dspace.lboro.ac.uk/) by the author and is made available under the following Creative Commons Licence conditions.

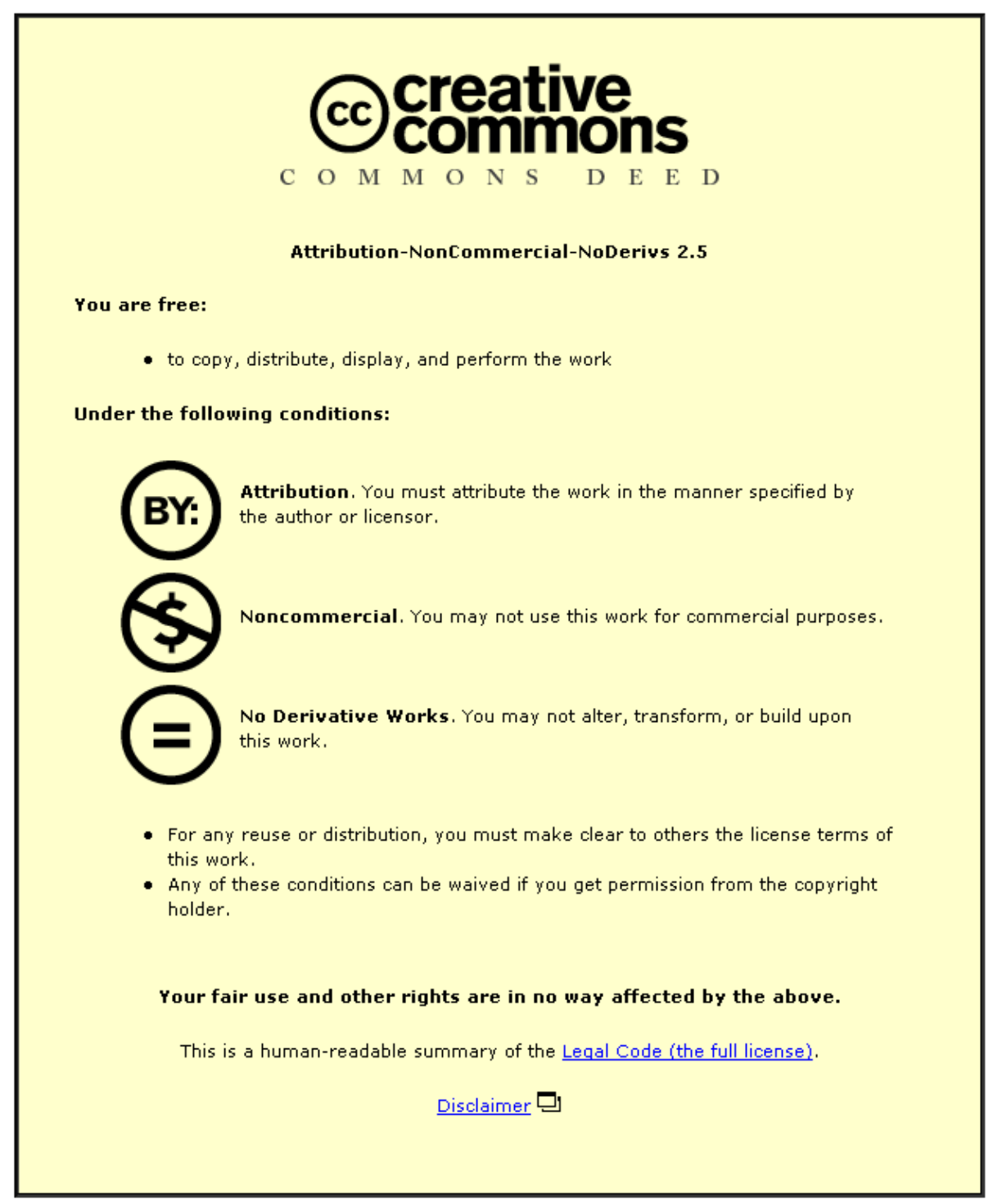

For the full text of this licence, please go to: http://creativecommons.org/licenses/by-nc-nd/2.5/ 


\title{
Joint Transceiver Design for MIMO Channel Shortening
}

\author{
Cenk Toker, Member, IEEE, Sangarapillai Lambotharan, Senior Member, IEEE, and \\ Jonathon A. Chambers, Senior Member, IEEE
}

\begin{abstract}
Channel shortening equalizers can be employed to shorten the effective impulse response of a long intersymbol interference (ISI) channel in order, for example, to decrease the computational complexity of a maximum-likelihood sequence estimator (MLSE) or to increase the throughput efficiency of an orthogonal frequency-division multiplexing (OFDM) transmission scheme. In this paper, the issue of joint transmitter-receiver filter design is addressed for shortening multiple-input multiple-output (MIMO) ISI channels. A frequency-domain approach is adopted for the transceiver design which is effectively equivalent to an infinite-length time-domain design. A practical space-frequency waterfilling algorithm is also provided. It is demonstrated that the channel shortening equalizer designed according to the time-domain approach suffers from an error-floor effect. However, the proposed techniques are shown to overcome this problem and outperform the time-domain channel shortening filter design. We also demonstrate that the proposed transceiver design can be considered as a MIMO broadband beamformer with constraints on the time-domain multipath length. Hence, a significant diversity gain could also be achieved by choosing strong eigenmodes of the MIMO channel. It is also found that the proposed frequency-domain methods have considerably low computational complexity as compared with their time-domain counterparts.
\end{abstract}

Index Terms-Broadband beamformer, channel shortening, equalization, joint transmitter-receiver design, multiple-input multiple-output (MIMO), waterfilling.

\section{INTRODUCTION}

I NTERSYMBOL interference (ISI) which is caused by the frequency-selective fading of a communication channel can be a major cause of performance degradation. In many schemes, such as IEEE 802.11g [2], HIPERLAN/2 [3], DAB [4], and DVB [5], spatial multiplexing [6], space-time block coding with time reversal (TR-STBC) [7] and [8], space-time orthogonal frequency-division multiplexing (ST-OFDM) [9], together with space-time trellis coding (STTC) [10] and [11], the use of either a maximum-likelihood sequence estimator (MLSE) or multicarrier modulation (MCM) is considered as an effective tool to tackle ISI. However, both methods have certain limitations related to the impulse response of the channel; in particular, the

Manuscript received February 1, 2006; revised October 19, 2006. This work was supported by EPSRC under Grant GR/S16775. The material in this paper was presented at the IEEE International Conference on Acoustics, Speech and Signal Processing, March 2005. The associate editor coordinating the review of this manuscript and approving it for publication was Prof. Martin Haardt.

C. Toker is with the Electrical and Electronics Engineering Department, Hacettepe University, 06800, Ankara, Turkey (e-mail: cenk.toker@ieee.org).

S. Lambotharan and J. A. Chambers are with the CDSP, Cardiff University, Cardiff CF24 3AA, U.K. (e-mail: LambotharanS@ cardiff.ac.uk; ChambersJ@ cardiff.ac.uk).

Digital Object Identifier 10.1109/TSP.2007.894231 implementation of these methods can be impractical when the channel has a relatively long impulse response.

The complexity of the MLSE for a long multipath channel can be prohibitively high because, for example, for a channel of length $n_{H}$ and a modulation size of $M$, the trellis for the Viterbi algorithm [12] requires $M^{n_{H}-1}$ states which can easily become very large with increasing $n_{H}$. The complexity would increase even further for multiple-input multiple-output (MIMO) channels.

For an MCM scheme, a cyclic prefix of length at least $\nu \geq$ $n_{H}-1$ has to be appended to each transmission frame of length $N$. Clearly, this reduces the throughput efficiency $N /(N+\nu)$ as the length of the channel increases.

These problems can be mitigated by channel shortening, a preprocessing technique for the MLSE or for MCM, which partially equalizes the channel so that the equalized channel has a shorter impulse response than that of the original channel, but does not reduce to a single spike (i.e., complete equalization). The length of the shortened channel is determined by the allowable cyclic prefix length for MCM or the allowable computational complexity for the MLSE.

In the literature, there have been many attempts to design a channel shortening filter using different criteria and objectives. Addressing the complexity issue of the MLSE, a linear front-end processor was proposed as early as the 1970s [13]. Later on, channel shortening was investigated for MCM in [14]. Channel shortening can be performed based on various criteria [15]-[17]. Martin et al. [15] provided a common framework for different types of channel shortening approaches which include those based on the following criteria: minimum mean-square error (MMSE) [13], [18], [19], and [20], maximum shortening signal-to-noise ratio (MSSNR) [21], minimum ISI (min-ISI) [22], [23], and [16], together with frequency domain methods such as per-tone-equalizer (PTEQ) [24] and filter-bank equalizer (TEQFB). Maximum geometric SNR (MGSNR) [14], maximum bit rate (MBR) [23] and [16], and maximum data rate (MDR) [17] are also other possible criteria for channel shortening filter design. Although almost all these works considered the channel shortening problem for single-input single-output (SISO) channels, the MMSE and MSSNR algorithms have also been used for MIMO channels, e.g., in [25] and [26].

When the channel state information (CSI) is available at the transmitter, it is possible to process the signal prior to transmission. This provides more degrees of freedom for equalization and noise suppression, and thereby enhances the performance. There are two major approaches for designing the transmitter and the receiver filters in such a scenario. The first approach 
is based on the time-domain design as in [27] and [28], and the second approach is based on the frequency-domain design as in [29], [30], and [31]. In this paper, the frequency-domain approach is adopted for joint optimization of the transmitter and receiver filters for MIMO channel equalization, based on the MMSE criterion. This is proven to be equivalent to an infinite-length time-domain equalizer design, such as [32]. Our work is different and more challenging than joint transceiver design for complete equalization, because, in addition to the transmitter and receiver filters, we also need to jointly optimize the target impulse response (TIR).

When the MMSE criterion is used, the aim is to minimize the distance between the equalized channel impulse response and a TIR of a prescribed length. It is found that although there exists a closed-form solution for the receiver filter, such a closedform solution is not available for the transmitter and the TIR filters. Instead, an iterative optimization method is necessary to determine these filters. For the transmitter filter, in particular, a space-frequency waterfilling algorithm is found to optimize the MMSE criterion. An extension of the algorithm in [33]-[35] is proposed as a practical way of implementing this waterfilling scheme. It is also demonstrated that, under certain transformations and conditions, the optimization problem can be cast into the optimization of a set of independent SISO subsystems, which significantly simplifies the design procedure.

We also determine the computational complexity of our proposed scheme and demonstrate that a significant saving in processing power is obtained for the computation of the proposed channel shortening filter parameters as compared with a timedomain channel shortening approach.

We also demonstrate that the proposed joint transceiver design for MIMO channel shortening can be generalized to a broadband MIMO beamformer with constraints on the maximum allowable multipath length. Hence, the proposed scheme provides spatial and frequency diversity for a frequency-selective channel while keeping the receiver complexity at a tolerable level.

The proposed technique will be very useful in particular for time-division duplex based wireless systems where the transmitter can obtain the CSI using the channel reciprocity. However, this does not impose any restrictions for the adaptation of our proposal to other systems where the CSI at the transmitter can be obtained through a feedback channel. The material in this paper was presented in part at the IEEE International Conference on Acoustics, Speech and Signal Processing, March 2005 [1].

The paper is organized as follows. Section II provides an overview of the MIMO channel shortening problem and develops necessary constraints for the design. In Section III, we design the receiver filter as a function of the transmitter filter and the TIR filter. In Section IV, certain transformations that diagonalize the whole system are first defined, and an iterative design procedure for the transmitter and the TIR filters is provided. In Section V, we design the channel shortening equalizer in the frequency domain without transmitter preprocessing. Section VI investigates and provides the computational complexity analysis of both the proposed methods and the time-domain equalizer design procedure. Section VII discusses the beamforming



Fig. 1. The structure of the channel shortening problem of a MIMO frequencyselective channel $\boldsymbol{H}(\omega)$, where the transmitter filter $\boldsymbol{C}(\omega)$, the receiver filter $\boldsymbol{Q}(\omega)$, and the target impulse response $\boldsymbol{B}(\omega)$ are jointly optimized to minimize the mean-square error (MSE) criterion $E\left\{\varepsilon^{H}(k) \varepsilon(k)\right\}$. The mutually uncorrelated data $\boldsymbol{d}(k)$ and noise $\boldsymbol{n}(k)$ have, respectively, power spectral density matrices $\boldsymbol{\Phi}_{d d}(\omega)$ and $\boldsymbol{\Phi}_{n n}(\omega)$.

property of the transmitter and receiver filters. Simulation results are provided in Section VIII and conclusions are drawn in Section IX.

Some of the common notations that are used throughout the paper are as follows. A scalar, a vector, and a matrix are denoted, respectively, by a lower case italic, a lower case boldface, and an upper case boldface letter. The time and frequency indices are $(k)$ and $(\omega)$. Complex conjugate, transpose, Hermitian transpose, convolution and trace operators are, respectively, represented by $(\cdot)^{*},(\cdot)^{T},(\cdot)^{H},(\cdot) *(\cdot)$, and $\operatorname{tr}\{\cdot\}$. The notation $n_{x}$ will be used for both the length of a filter and the dimension of a vector.

\section{Problem Statement}

Consider a frequency-selective MIMO channel with $n_{T}$ transmit and $n_{R}$ receive antennas. The length of the impulse response of all subchannels is assumed to be $n_{H}$. The primary goal of a channel shortening filter is to reduce this channel impulse response length to a desired length $n_{b}$, while taking certain criteria and constraints into consideration. The most widely examined criterion due to its noise immunity is MMSE which motivates its use in this study. To proceed with our development, along with the physical channel, and the transmitter and receiver filters, as seen on the upper branch of Fig. 1, a second virtual path is provided in the lower branch to represent the desired shortened channel impulse response. This branch is composed of a TIR with a delay $m$. In addition to the transmitter and receiver filters, the TIR filter coefficients and the associated delay are also design parameters to be optimized by the algorithm. The length of the TIR, $n_{b}$, is not a design parameter but a given fixed integer. It will be shown later that the proposed design is insensitive to the delay $m$, in contrast to the conventional channel shortening/equalization methods, for example [25], where the delay significantly affects the performance.

The design will be performed in the frequency domain, therefore the notation $\boldsymbol{C}(\omega), \boldsymbol{H}(\omega), \boldsymbol{Q}(\omega)$, and $\boldsymbol{B}(\omega)$ will be used, respectively, for the frequency responses of the transmitter filter, the channel, the receiver filter, and the TIR filter at a particular frequency $\omega$. Note that, since the design is for a MIMO channel, all these filters are defined, respectively, as matrices with corresponding dimensions $n_{T} \times n_{i}, n_{R} \times n_{T}, n_{i} \times n_{R}$, and $n_{i} \times n_{i}$. Here, $n_{i}$ is the number of the parallel input data streams in $\boldsymbol{d}(k)$. The signal at the output of the MIMO channel is

$$
\boldsymbol{y}(k)=\boldsymbol{H}(k) * \boldsymbol{C}(k) * \boldsymbol{d}(k)+\boldsymbol{n}(k)
$$


where the zero-mean circularly symmetric additive Gaussian noise is represented by an $n_{R} \times 1$ vector $\boldsymbol{n}(k)$. Both the data and the noise can be colored with power spectral density matrices $\boldsymbol{\Phi}_{d d}(\omega)$ and $\boldsymbol{\Phi}_{n n}(\omega)$. However, the data and noise are assumed to be mutually uncorrelated. Also, one can write the TIR in open form as

$$
\boldsymbol{B}\left(q^{-1}\right)=\boldsymbol{B}_{0}+\boldsymbol{B}_{1} q^{-1}+\cdots+\boldsymbol{B}_{n_{b}-1} q^{-\left(n_{b}-1\right)}
$$

where $q^{-1}$ is the unit delay operator and the $n_{i} \times n_{i}$ matrix taps $\boldsymbol{B}_{i}, i=0, \ldots, n_{b}-1$ are coefficients of the TIR filter.

The $n_{i} \times 1$ error signal vector $\varepsilon(k)$ is defined as the difference between the outputs of the TIR and the receiver filter, i.e., the difference between the desired signal $\boldsymbol{z}(k)$ and the (channel shortening) equalized signal $\hat{\boldsymbol{z}}(k)$

$$
\begin{aligned}
\varepsilon(k) & =\boldsymbol{z}(k)-\hat{\boldsymbol{z}}(k) \\
& =\left[q^{-m} \boldsymbol{B}(k)-\boldsymbol{Q}(k) * \boldsymbol{H}(k) * \boldsymbol{C}(k)\right] * \boldsymbol{d}(k)-\boldsymbol{Q}(k) * \boldsymbol{n}(k) .
\end{aligned}
$$

Hence, the mean-square error (MSE) is defined as follows:

$$
J(\boldsymbol{Q}(k), \boldsymbol{C}(k), \boldsymbol{B}(k))=\operatorname{tr} E\left\{\boldsymbol{\varepsilon}(k) \varepsilon^{H}(k)\right\} .
$$

Substituting (3) into (4) and applying Parseval's formula [36], we can write the MSE in the frequency domain as

$$
\begin{gathered}
J(\boldsymbol{Q}(\omega), \boldsymbol{C}(\omega), \boldsymbol{B}(\omega)) \\
=\frac{1}{2 \pi} \operatorname{tr} \int_{-\pi}^{\pi}\left\{\left[e^{-j \omega m} \boldsymbol{B}(\omega)-\boldsymbol{Q}(\omega) \boldsymbol{H}(\omega) \boldsymbol{C}(\omega)\right](\omega)\right. \\
\boldsymbol{\Phi}_{d d}\left[e^{-j \omega m} \boldsymbol{B}(\omega)-\boldsymbol{Q}(\omega) \boldsymbol{H}(\omega) \boldsymbol{C}(\omega)\right]^{H} \\
\left.+\boldsymbol{Q}(\omega) \boldsymbol{\Phi}_{n n}(\omega) \boldsymbol{Q}^{H}(\omega)\right\} d \omega .
\end{gathered}
$$

It can be shown that, without any constraints, the minimization of (5) will yield the trivial solution $B=Q=0$ and $\boldsymbol{C}=\infty$. The first condition is meaningless because it blocks the transmission and the data cannot be conveyed through the equalized channel. The second condition means infinite transmit power which is practically impossible. Therefore, certain constraints should be imposed on the filters. In our study, we impose an orthogonality constraint on the TIR and a limited power constraint on the transmitter filter as follows:

$$
\begin{aligned}
& \frac{1}{2 \pi} \int_{-\pi}^{\pi} \boldsymbol{U}^{H}(\omega) \boldsymbol{B}(\omega) \boldsymbol{B}^{H}(\omega) \boldsymbol{U}(\omega) d \omega=\boldsymbol{I} \\
& \frac{1}{2 \pi} \operatorname{tr} \int_{-\pi}^{\pi} \boldsymbol{C}(\omega) \boldsymbol{\Phi}_{d d}(\omega) \boldsymbol{C}^{H}(\omega) d \omega=P_{o}
\end{aligned}
$$

where $\boldsymbol{U}(\omega)$ is an $n_{i} \times n_{i}$ unitary matrix that will be described in Section IV-A and $P_{o}$ is some finite power level. Therefore, the optimization problem becomes the minimization of (5) under constraints (6a) and (6b).

For notational simplicity, when there is no ambiguity, the parenthesis $(\omega)$ will be dropped in the sequel for filters represented in the frequency domain. However, for the time-domain representations, the parenthesis $(k)$ will be retained.

\section{RECEIVER FILTER}

The optimum receiver filter minimizing the MSE in (5) can be found by employing the principle of orthogonality, i.e.,

$$
E\left\{\varepsilon(k) \boldsymbol{y}^{H}(k)\right\}=\mathbf{0} .
$$

Substituting (1) and (3) into (7) and employing Parseval's formula, we obtain

$$
\begin{aligned}
\frac{1}{2 \pi} \int_{-\pi}^{\pi}\left\{e^{-j \omega m} \boldsymbol{B} \boldsymbol{\Phi}_{d d} \boldsymbol{C}^{H} \boldsymbol{H}^{H}\right. \\
\left.-\boldsymbol{Q}\left(\boldsymbol{H} C \boldsymbol{\Phi}_{d d} \boldsymbol{C}^{H} \boldsymbol{H}^{H}+\boldsymbol{\Phi}_{n n}\right)\right\} d \omega=\mathbf{0} .
\end{aligned}
$$

In order to satisfy the equality, it is adequate to make the integrand equal to zero, yielding

$$
\begin{aligned}
\boldsymbol{Q}_{\mathrm{opt}} & =e^{-j \omega m} \boldsymbol{B} \boldsymbol{\Phi}_{d d} \boldsymbol{C}^{H} \boldsymbol{H}^{H}\left(\boldsymbol{H} C \boldsymbol{\Phi}_{d d} \boldsymbol{C}^{H} \boldsymbol{H}^{H}+\boldsymbol{\Phi}_{n n}\right)^{-1} \\
& =e^{-j \omega m} \boldsymbol{B}\left(\boldsymbol{\Phi}_{d d}^{-1}+\boldsymbol{C}^{H} \boldsymbol{H}^{H} \boldsymbol{\Phi}_{n n}^{-1} \boldsymbol{H} C\right)^{-1} \boldsymbol{C}^{H} \boldsymbol{H}^{H} \boldsymbol{\Phi}_{n n}^{-1}
\end{aligned}
$$

where the matrix inversion lemma (MIL) ${ }^{1}$ is used in the second line. It is interesting to note the whitening matched filter front-end $\boldsymbol{H}^{H} \boldsymbol{\Phi}_{n n}^{-1}$ appears in $\boldsymbol{Q}_{\text {opt }}$.

By substituting (9) into (5) and utilizing the MIL, we obtain the MSE as a function of only $\boldsymbol{C}$ and $\boldsymbol{B}$

$$
J(\boldsymbol{C}, \boldsymbol{B})=\frac{1}{2 \pi} \operatorname{tr} \int_{-\pi}^{\pi} \boldsymbol{B}\left[\boldsymbol{\Phi}_{d d}^{-1}+\boldsymbol{C}^{H} \boldsymbol{H}^{H} \boldsymbol{\Phi}_{n n}^{-1} \boldsymbol{H} C\right]^{-1} \boldsymbol{B}^{H} d \omega .
$$

Remark 1: Observe that $\boldsymbol{Q}_{\mathrm{opt}}$ is directly related to $B$, hence, the constraint in (6a) also forces the receiver filter to be nonzero, avoiding a trivial solution.

Remark 2: Another way of finding the optimum receiver filter is actually enforcing the integral in (8) to be equal to zero, which results in a different type of filter. Details of such a design can be found in [37]-[39].

\section{TRANSMITTER AND TIR OPTIMIZATION}

Minimization of (10) is not as straightforward as the receiver filter design due to the involvement of matrices and the constraints in (6). The procedure will be greatly simplified if the problem can be expressed in terms of a number of independent parallel SISO subsystems. This can be achieved through certain transformations.

\section{A. Transformations}

First, consider the eigendecomposition of the spectral density matrix of the data process $\boldsymbol{d}(k)$

$$
\boldsymbol{\Phi}_{d d}(\omega)=\boldsymbol{U}(\omega) \boldsymbol{K}_{d d}(\omega) \boldsymbol{U}^{H}(\omega)
$$

where $\boldsymbol{U}(\omega)$ is an $n_{i} \times n_{i}$ unitary matrix and the eigenvalues $\kappa_{i}(\omega), i=1, \ldots, n_{i}$ of $\Phi_{d d}(\omega)$ are the entries of the $n_{i} \times n_{i}$

${ }^{1}$ Matrix Inversion Lemma: $(\boldsymbol{A}+\boldsymbol{B C} \boldsymbol{D})^{-1}=A^{-1}-A^{-1} \boldsymbol{B}\left(\boldsymbol{D} A^{-1} \boldsymbol{B}+\right.$ $\left.\boldsymbol{C}^{-1}\right)^{-1} \boldsymbol{D} \boldsymbol{A}^{-1}$ 
diagonal matrix $\boldsymbol{K}_{d d}(\omega)$. The matrix $\boldsymbol{\Phi}_{d d}(\omega)$, or equivalently, $K_{d d}(\omega)$, can be rank deficient for a particular frequency $\omega$. Although we assume that the matrix $\boldsymbol{\Phi}_{d d}(\omega)$ is full rank for the sake of notational simplicity, this work can be extended to the rank deficient case by simply replacing the parameter $n_{i}$ by the rank of $\boldsymbol{\Phi}_{d d}(\omega)$ without affecting the logic of the analysis presented here. In fact, the waterfilling algorithm provided in Section IV-D automatically performs this task.

The second decomposition that will be used is

$$
\begin{aligned}
& \boldsymbol{H}^{H}(\omega) \boldsymbol{\Phi}_{n n}^{-1}(\omega) \boldsymbol{H}(\omega) \\
& \quad=\left[\begin{array}{ll}
\boldsymbol{V}(\omega) & \overline{\boldsymbol{V}}(\omega)
\end{array}\right]\left[\begin{array}{cc}
\boldsymbol{\Lambda}_{n n}^{-1}(\omega) & \mathbf{0} \\
\mathbf{0} & \mathbf{0}
\end{array}\right]\left[\begin{array}{l}
\boldsymbol{V}^{H}(\omega) \\
\overline{\boldsymbol{V}}^{H}(\omega)
\end{array}\right]
\end{aligned}
$$

where the diagonal matrix $\Lambda_{n n}^{-1}(\omega)$, of size $P \times P$, contains the positive eigenvalues $\lambda_{i}^{-1}(\omega)$ of $\boldsymbol{H}^{H}(\omega) \boldsymbol{\Phi}_{n n}^{-1}(\omega) \boldsymbol{H}(\omega)$ and the $n_{T} \times P$ unitary matrix $V(\omega)$ contains the corresponding eigenvectors. Similarly, the $n_{T} \times\left(n_{T}-P\right)$ unitary matrix, $\overline{\boldsymbol{V}}(\omega)$ spans the null space of $\boldsymbol{H}^{H}(\omega) \boldsymbol{\Phi}_{n n}^{-1}(\omega) \boldsymbol{H}(\omega)$. The parameter $P$ for a particular frequency $\omega$ is the number of subchannels (i.e., nonzero eigenmodes) that can convey information in the equivalent system after diagonalization, and is defined as $P=$ $\min \left\{n_{T}, n_{R}, \operatorname{rank}\{\boldsymbol{H}(\omega)\}\right\}$. The value of $P$ can be frequency dependent. Without loss of generality, the entries of $\boldsymbol{\Lambda}_{n n}(\omega)$ are assumed to be ordered as follows:

$$
0<\lambda_{1}(\omega) \leq \lambda_{2}(\omega) \leq \cdots \leq \lambda_{P}(\omega)
$$

Remark 3: Note that these decompositions are carried out for each distinct frequency $\omega$. In a practical application, the frequency range $[0,2 \pi)$ is partitioned into $N$ bins with $\omega_{k}=$ $2 \pi k / N, k=0, \ldots, N-1$, and the decompositions are evaluated for each particular $\omega_{k}$.

We now define two matrices, $\boldsymbol{F}(\omega)$ and $\boldsymbol{B}_{d}(\omega)$, of size $P \times n_{i}$ and $n_{i} \times n_{i}$, respectively, such that

$$
\begin{aligned}
& \boldsymbol{C}(\omega) \triangleq \boldsymbol{V}(\omega) \boldsymbol{F}(\omega) \boldsymbol{U}^{H}(\omega) \\
& \boldsymbol{B}(\omega) \triangleq \boldsymbol{U}(\omega) \boldsymbol{B}_{d}(\omega) \boldsymbol{U}^{H}(\omega) .
\end{aligned}
$$

We will prove later that for optimum $\boldsymbol{C}(\omega)$ and $\boldsymbol{B}(\omega)$, the matrices $\boldsymbol{F}(\omega)$ and $\boldsymbol{B}_{d}(\omega)$ are diagonal. Using the decompositions (11), (12), and the definitions (14), the optimum receiver filter in (9) can be rewritten as

$$
\begin{aligned}
\boldsymbol{Q}_{\mathrm{opt}} & =e^{-j \omega m} \boldsymbol{U} B_{d}\left(\boldsymbol{K}_{d d}^{-1}+\boldsymbol{F}^{H} \boldsymbol{\Lambda}_{n n}^{-1} \boldsymbol{F}\right)^{-1} \boldsymbol{F}^{H} \boldsymbol{V}^{H} \boldsymbol{H}^{H} \boldsymbol{\Phi}_{n n}^{-1} \\
& =e^{-j \omega m} \boldsymbol{U} B_{d} \boldsymbol{G} V^{H} \boldsymbol{H}^{H} \boldsymbol{\Phi}_{n n}^{-1}
\end{aligned}
$$

where $\boldsymbol{G}=\left(\boldsymbol{K}_{d d}^{-1}+\boldsymbol{F}^{H} \boldsymbol{\Lambda}_{n n}^{-1} \boldsymbol{F}\right)^{-1} \boldsymbol{F}^{H}$.

When the receiver filter is set to its optimum condition, as in (15), the receiver filter output can be written as

$$
\hat{\boldsymbol{z}}(\omega)=\boldsymbol{Q}_{\mathrm{opt}}(\omega) \boldsymbol{H}(\omega) \boldsymbol{C}(\omega) \boldsymbol{d}(\omega)+\boldsymbol{Q}_{\mathrm{opt}}(\omega) \boldsymbol{n}(\omega)
$$

where $\hat{\boldsymbol{z}}(\omega), \boldsymbol{d}(\omega)$, and $\boldsymbol{n}(\omega)$ are, respectively, the frequencydomain representations of the signal at the receiver filter output, the data, and the noise.



Fig. 2. The equivalent system in the frequency domain after the transformations $\mathcal{U}(\omega)$ and $V(\omega)$. The transformed data and noise processes are spatially white and the original MIMO channel is replaced with an ideal channel which is an identity matrix.

Substituting (14) and (15) into (16), and using the property $V^{H} \boldsymbol{H}^{H} \boldsymbol{\Phi}_{n n}^{-1} \boldsymbol{H} V=\boldsymbol{\Lambda}_{n n}^{-1}(\boldsymbol{\omega})$ from (12) and the definition

$$
W(\omega) \triangleq \boldsymbol{G}(\omega) \boldsymbol{\Lambda}_{n n}^{-1}(\omega)
$$

we can write the error signal at frequency $\omega$ as

$$
\begin{aligned}
\varepsilon(\omega)= & \boldsymbol{z}(\omega)-\hat{\boldsymbol{z}}(\omega) \\
= & \boldsymbol{U} e^{-j \omega m}\left[\boldsymbol{B}_{d}-\boldsymbol{B}_{d} \boldsymbol{W} \boldsymbol{F}\right] \boldsymbol{U}^{H} \boldsymbol{d}(\omega) \\
& -e^{-j \omega m} \boldsymbol{U} \boldsymbol{B}_{d} \boldsymbol{G V}^{H} \boldsymbol{H}^{H} \boldsymbol{\Phi}_{n n}^{-1} \boldsymbol{n}(\omega) .
\end{aligned}
$$

Hence, with the substitution for the optimum receiver filter in (15), the MSE in (5) can be written as

$$
\begin{gathered}
J(\boldsymbol{Q}, \boldsymbol{C}, \boldsymbol{B})=\frac{1}{2 \pi} \operatorname{tr} \int_{-\pi}^{\pi}\left\{\boldsymbol{U} \boldsymbol{B}_{d}[\boldsymbol{I}-\boldsymbol{W} \boldsymbol{F}] \boldsymbol{U}^{H} \boldsymbol{\Phi}_{d d} \boldsymbol{U}\right. \\
\left.[\boldsymbol{I}-\boldsymbol{W} \boldsymbol{F}]^{H} \boldsymbol{B}_{d}^{H} \boldsymbol{U}^{H}+\boldsymbol{U} \boldsymbol{B}_{d} \boldsymbol{G} \boldsymbol{V}^{H} \boldsymbol{H}^{H} \boldsymbol{\Phi}_{n n}^{-1} \boldsymbol{H} \boldsymbol{V} \boldsymbol{G}^{H} \boldsymbol{B}_{d}^{H} \boldsymbol{U}^{H}\right\} d \omega \\
=\frac{1}{2 \pi} \operatorname{tr} \int_{-\pi}^{\pi}\left\{\left[\boldsymbol{B}_{d}-\boldsymbol{B}_{d} \boldsymbol{W} \boldsymbol{F}\right] \boldsymbol{K}_{d d}\left[\boldsymbol{B}_{d}-\boldsymbol{B}_{d} \boldsymbol{W} \boldsymbol{F}\right]^{H}\right. \\
\left.\quad+\boldsymbol{B}_{d} \boldsymbol{W} \boldsymbol{\Lambda}_{n n} \boldsymbol{W}^{H} \boldsymbol{B}_{d}^{H}\right\} d \omega
\end{gathered}
$$

where in the second line we used (17) as well as the property $\operatorname{tr}\{\boldsymbol{A} \boldsymbol{B}\}=\operatorname{tr}\{\boldsymbol{B} \boldsymbol{A}\}$ for appropriate dimensions to eliminate the matrices $\boldsymbol{U}$ and $\boldsymbol{U}^{H}$.

Now, using the analogy between Fig. 1 and (5), we can express the transformed system as in Fig. 2 considering (18), and by defining the transformed data and noise signal vectors $\tilde{\boldsymbol{d}}(\omega)$ and $\tilde{\boldsymbol{n}}(\omega)$, with power spectral densities $\boldsymbol{K}_{d d}(\omega)$ and $\Lambda_{n n}(\omega)$.

In summary, the transmitter, receiver, and TIR filters can be expressed in the transformed domain with the following pairs:

$$
\begin{aligned}
\boldsymbol{C}(\omega) & \leftrightarrow \boldsymbol{F}(\omega) \\
\boldsymbol{Q}(\omega) & \leftrightarrow \boldsymbol{B}_{d}(\omega) \boldsymbol{W}(\omega) \\
\boldsymbol{B}(\omega) & \leftrightarrow \boldsymbol{B}_{d}(\omega) .
\end{aligned}
$$

After substituting the definitions in (14) into the MSE expression in (10), the optimization problem reduces to determining the optimum $\boldsymbol{F}$ and $\boldsymbol{B}_{d}$ to minimize the MSE

$$
J\left(\boldsymbol{F}, \boldsymbol{B}_{d}\right)=\frac{1}{2 \pi} \operatorname{tr} \int_{-\pi}^{\pi} \boldsymbol{B}_{d}\left[\boldsymbol{K}_{d d}^{-1}+\boldsymbol{F}^{H} \boldsymbol{\Lambda}_{n n}^{-1} \boldsymbol{F}\right]^{-1} \boldsymbol{B}_{d}^{H} d \omega
$$




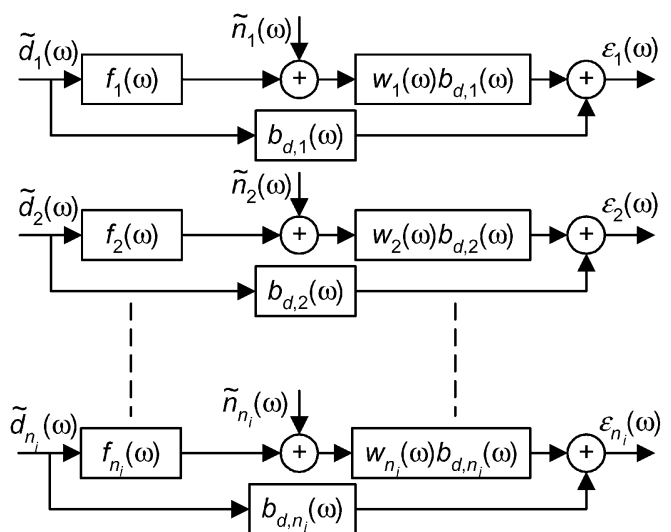

Fig. 3. The equivalent system in the form of uncoupled parallel SISO subchannels. The new set of data and the noise are white, and the subchannels are AGN with unity taps.

under the following constraints obtained by substituting (14) into (6):

$$
\begin{aligned}
& \frac{1}{2 \pi} \int_{-\pi}^{\pi} \boldsymbol{B}_{d}(\omega) \boldsymbol{B}_{d}^{H}(\omega) d \omega=\boldsymbol{I} \\
& \frac{1}{2 \pi} \operatorname{tr} \int_{-\pi}^{\pi} \boldsymbol{F}(\omega) \boldsymbol{K}_{d d}(\omega) \boldsymbol{F}^{H}(\omega) d \omega \leq P_{o} .
\end{aligned}
$$

Remark 4: Note the similarity of (20a) to the orthogonality constraint of [25].

\section{B. Diagonalization}

In this subsection, we will prove that under certain conditions the optimum transformed filters $\boldsymbol{B}_{d, \text { opt }}(\omega)$ and $\boldsymbol{F}_{\text {opt }}(\omega)$ are diagonal with entries $b_{d, k}(\omega), k=1, \ldots, n_{i}$, and $f_{k}(\omega)$, $k=1, \ldots, P$. This will allow us to express the optimization problem as independent parallel SISO subsystems as demonstrated in Fig 3. Notice that the effective channel has become a unity tap with additive Gaussian noise $\tilde{n}_{i}(k)$, i.e., the $i$ th entry of the vector $\tilde{\boldsymbol{n}}(k)$.

1) Optimum TIR Design: In order to prove that the optimum $\boldsymbol{B}_{d}$ is diagonal, we first state the following theorem.

Theorem 1: The matrix $\left[\boldsymbol{K}_{d d}^{-1}+\boldsymbol{F}^{H} \boldsymbol{\Lambda}_{n n}^{-1} \boldsymbol{F}\right]^{-1}$ is diagonal for optimality.

Proof: See Appendix I.

Theorem 2: The optimum TIR, $B_{d, \mathrm{opt}}(\omega)$, is diagonal.

Proof: See Appendix II.

Due to the diagonality of $\boldsymbol{B}_{d, \mathrm{opt}}(\omega)$, the TIR can be expressed as independent SISO sub-TIRs. Moreover, the orthogonality constraint in (20a) can be rewritten as

$$
\frac{1}{2 \pi} \int_{-\pi}^{\pi}\left|b_{d, k}(\omega)\right|^{2} d \omega=1, \quad k=1, \ldots, n_{i} .
$$

2) Optimum Transmitter Filter Design: Since CSI is available at the transmitter, it is possible to activate only the nonzero eigenmodes of the diagonalized channels, i.e., $n_{i}<P$, in order not to waste transmit power and to prevent information loss.
Theorem 3: The optimum transformed transmitter filter $F_{\text {opt }}(\omega)$ is diagonal for the ordering

$$
\kappa_{1}(\omega)\left|b_{d, 1}(\omega)\right|^{2} \geq \kappa_{2}(\omega)\left|b_{d, 2}(\omega)\right|^{2} \geq \cdots \geq \kappa_{n_{i}}(\omega)\left|b_{d, n_{i}}(\omega)\right|^{2}
$$

Proof: See Appendix III.

Using the diagonality of $\boldsymbol{F}(\omega)$, we can rewrite the constraint on transmit power in (20b) as

$$
\frac{1}{2 \pi} \sum_{k=1}^{n_{i}} \int_{-\pi}^{\pi} \kappa_{k}(\omega)\left|f_{k}(\omega)\right|^{2} d \omega \leq P_{o}
$$

\section{Joint Transmitter Filter and TIR Optimization}

With the diagonality of the transformed transmitter filter and TIR structures, we can write the MSE expression in (19) as the sum of a set of independent subchannel MSEs

$$
J\left(\boldsymbol{F}, \boldsymbol{B}_{d}\right)=\frac{1}{2 \pi} \sum_{k=1}^{n_{i}} \int_{-\pi}^{\pi}\left|b_{d, k}\right|^{2}\left(\frac{\kappa_{k} \lambda_{k}}{\lambda_{k}+\kappa_{k}\left|f_{k}\right|^{2}}\right) d \omega .
$$

Together with the constraints in (21) and (23), the cost function in (24) does not provide a closed-form solution for the transmitter filter and TIR expressions. However, as provided by the following theorem, given that the cost function $J\left(\boldsymbol{F}, \boldsymbol{B}_{d}\right)$ is convex with respect to the variable sets $\mathcal{B}(\omega)=\left\{b_{d, k}(\omega)\right.$, $\left.k=1, \ldots, n_{i}\right\}$, and $\mathcal{F}(\omega)=\left\{\left|f_{k}(\omega)\right|^{2}, k=1, \ldots, n_{i}\right\}$, the optimization can be carried out in an iterative manner. That is, in the first step of a particular iteration, the transmitter filter is calculated while the TIR which was calculated in the previous iteration is kept fixed. Similarly, in the second step, the TIR is calculated assuming that the transmitter filter is fixed. These alternating iterations are carried out until the reduction in MSE becomes negligible.

Theorem 4: The integrand of (24) is jointly convex in $\mathcal{B}(\omega)$ and $\mathcal{F}(\omega)$.

Proof: This can easily be proven using Jensen's inequality [40], which states that a function ${ }^{2} f: \mathbb{C}^{n} \times \mathbb{R}_{+,\{0\}}^{n_{i}} \rightarrow \mathbb{R}_{+,\{0\}}$ is convex if the domain of $f(\operatorname{dom} f)$ is a convex set and if $\forall$ $x, y \in \operatorname{dom} f$, the following inequality holds:

$$
f(\theta x+(1-\theta) y) \leq \theta f(x)+(1-\theta) f(y)
$$

where $\theta, 0 \leq \theta \leq 1$ is a real scalar. The function to be investigated is

$$
\zeta(\{\mathcal{B}, \mathcal{F}\})=\sum_{k=1}^{n_{i}}\left|b_{d, k}\right|^{2}\left(\frac{\kappa_{k} \lambda_{k}}{\lambda_{k}+\kappa_{k}\left|f_{k}\right|^{2}}\right) .
$$

The domain of $\zeta(\{\mathcal{B}, \mathcal{F}\})$ is convex since $b_{d, k}(\omega) \in \mathbb{C}^{n_{i}}$ and $\left|f_{k}(\omega)\right|^{2} \in \mathbb{R}_{+,\{0\}}^{n_{i}}$. Substituting $\zeta(\{\mathcal{B}, \mathcal{F}\})$ into (25) as

$$
\begin{aligned}
& \sum_{k=1}^{n_{i}}\left|\theta b_{d, k}+(1-\theta) b_{d, k}^{\prime}\right|^{2}\left(\frac{\kappa_{k} \lambda_{k}}{\lambda_{k}+\kappa_{k}\left(\theta\left|f_{k}\right|^{2}+(1-\theta)\left|f_{k}^{\prime}\right|^{2}\right)}\right) \\
& \leq \sum_{k=1}^{n_{i}} \theta\left|b_{d, k}\right|^{2}\left(\frac{\kappa_{k} \lambda_{k}}{\lambda_{k}+\kappa_{k}\left|f_{k}\right|^{2}}\right) \\
& \quad+\sum_{k=1}^{n_{i}}(1-\theta)\left|b_{d, k}^{\prime}\right|^{2}\left(\frac{\kappa_{k} \lambda_{k}}{\lambda_{k}+\kappa_{k}\left|f_{k}^{\prime}\right|^{2}}\right)
\end{aligned}
$$

${ }^{2} \mathbb{R}_{+,\{0\}}$ is the set of positive real numbers also including the zero element. 
where $b_{d, k}^{\prime}(\omega) \in \mathbb{C}^{n_{i}}$ and $\left|f_{k}^{\prime}(\omega)\right|^{2} \in \mathbb{R}_{+,\{0\}}^{n_{i}}$. The inequality can be verified by expanding and simplifying (26), concluding the proof of Theorem 4. For further details see [39].

Corollary 1: Convexity of the integrand of (24) guarantees the convexity of the integration itself [40], hence, there exists a single minimum of the MSE with respect to $b_{d, k}(\omega)$ and $\left|f_{k}(\omega)\right|^{2}$. Therefore, the convexity of the iterative method is also guaranteed. Simulations revealed that three to four iterations are adequate to converge to the vicinity of the minimum point.

Remark 5: The MSE in (24) depends on $n_{i}$ independent sets of variables $b_{d, k}(\omega)$ and $\left|f_{k}(\omega)\right|^{2}$. As proven in the preceding theorem, each set is convex within itself. Hence, the global minimum can be attained by independent minimizations.

Remark 6: Note that the delay term $e^{-j \omega m}$ does not appear in (24) which means that the optimization is independent of the delay $m$ in contrast to the finite-length channel shortening filter design, for example [25], where the delay parameter $m$ - together with the target impulse response-is used to select a submatrix in a square window centered on the main diagonal of a certain covariance matrix. Observe that the frequency-domain approach in this paper is effectively equivalent to an infinite-length filter design in the time domain. When the filter length is finite, the covariance matrix is Hermitian symmetric but not Toeplitz. It can be shown that as the filter length increases, the covariance matrix approaches a Toeplitz form [32], [39]. In this case, since the covariance matrix is Toeplitz, all the submatrices in a square window chosen along the main diagonal by the delay parameter $m$ will be the same, meaning that the design becomes insensitive to the delay $m$.

\section{Transmitter Filter Design (Space-Frequency Waterfilling)}

In the iterative method, the optimization of the transmitter filter is first investigated assuming the TIR is known a priori. The following theorem summarizes the transmitter filter design.

Theorem 5: For the optimum transmitter filter $\boldsymbol{F}_{\text {opt }}(\omega)=$ $\operatorname{diag}\left\{f_{0}(\omega), f_{1}(\omega), \ldots, f_{n_{i}-1}(\omega)\right\}$, the magnitude response should be

$$
\left|f_{k}(\omega)\right|^{2}=\max \left\{\gamma \sqrt{\left|b_{d, k}(\omega)\right|^{2} \frac{\lambda_{k}(\omega)}{\kappa_{k}(\omega)}}-\frac{\lambda_{k}(\omega)}{\kappa_{k}(\omega)}, 0\right\}
$$

for $k=1, \ldots, P$ where $\gamma$ is chosen to satisfy the power constraint in (23).

Proof: Construct the Lagrangian by adding the power constraint (23) to the MSE (24) with the variable $1 / \gamma^{2}$. The result in (27) is obtained by differentiating the Lagrangian cost function with respect to $\left|f_{k}(\omega)\right|^{2}$, equating it to zero and imposing $\left|f_{k}(\omega)\right|^{2} \geq 0$.

Remark 7: Note that (27) is a waterfilling algorithm which distributes the total transmit power among each subchannel and frequency so as to minimize the MSE criterion. Also note that (27) only determines the magnitude response of the transmitter filter whereas the phase is not required for optimality, and it can be chosen arbitrarily for a general solution. However, the phase could also be used to manipulate the impulse response of the filters, see for example [41]. The phase could also be exploited to obtain a stable infinite impulse response (IIR) realization.
Assuming that the frequency range $[0,2 \pi)$ is divided into $N$ bins, the following algorithm which is an extension of [33]-[35] finds the exact solution of the space-frequency waterfilling in (27) and the waterlevel $\gamma$ in at most NP iterations, in contrast to [29]-[31], where the number of iterations can be unbounded. In our case, the total energy is distributed among both space and frequency jointly, rather than only in the frequency domain. For more details see [39].

Algorithm: Let the set of ordered pairs $(k, l) \forall k, l$ be sorted in ascending order according to the quotient $q(j)=\alpha_{k, l} / \beta_{k, l}$ where $\alpha_{k, l}=\lambda_{k}\left(\omega_{l}\right) / \kappa_{k}\left(\omega_{l}\right)$ and

$$
\beta_{k, l}=\sqrt{\left|b_{d, k}\left(\omega_{l}\right)\right|^{2} \lambda_{k}\left(\omega_{l}\right) / \kappa_{k}\left(\omega_{l}\right)}
$$

$k=1, \ldots, P, l=0, \ldots, N-1$, and $j=l P+k \in \mathcal{S}=$ $\{1, \ldots, N P\}$.

1) Calculate the waterlevel $\gamma$ according to

$$
\gamma=\frac{P_{o}+\sum_{j \in \mathcal{S}} \alpha_{k, l}}{\sum_{j \in \mathcal{S}} \beta_{k, l}} .
$$

2) If for the last element of the set $\mathcal{S},\left|f_{k}\left(\omega_{l}\right)\right|^{2}$ takes a negative value, i.e., $\left|f_{k}\left(\omega_{l}\right)\right|^{2}=\gamma \beta_{k, l}-\alpha_{k, l}<0$, then remove the pair $(k, l)$ from $\mathcal{S}$ and move the cursor to the position above and go to Step 2 .

3) If no negative value is left, stop the loop and calculate the final power levels of each bin according to (27) using the waterlevel obtained from the last iteration.

\section{E. TIR Design}

The second step of the iterative optimization involves the TIR design, under the assumption that the transmitter filter is known a priori. We can write the transformed TIR $b_{d, k}(\omega)$ for each subchannel in a vector form as

$$
\begin{aligned}
b_{d, k}(\omega) & =\tilde{b}_{d, k, 0}+\cdots+\tilde{b}_{d, k, n_{b}-1} e^{-j \omega\left(n_{b}-1\right)} \\
& =\left[\begin{array}{lll}
\tilde{b}_{d, k, 0} & \cdots & \tilde{b}_{d, k, n_{b}-1}
\end{array}\right]\left[\begin{array}{c}
1 \\
\vdots \\
e^{-j \omega\left(n_{b}-1\right)}
\end{array}\right] \\
& =\tilde{\boldsymbol{b}}_{d, k} \boldsymbol{\Omega} .
\end{aligned}
$$

Substituting this definition into (24) and performing the numerical evaluation of the resulting integral, the MSE for the $k$ th subchannel becomes

$$
J_{k}\left(b_{d, k}\right)=\tilde{\boldsymbol{b}}_{d, k} \boldsymbol{R}_{k} \tilde{\boldsymbol{b}}_{d, k}^{H}
$$

where the autocorrelation matrix $\boldsymbol{R}_{k}$ is

$$
\left[\boldsymbol{R}_{k}\right]_{m, n}=\frac{1}{2 \pi} \int_{-\pi}^{\pi} e^{-j \omega(m-n)}\left(\frac{\kappa_{k} \lambda_{k}}{\lambda_{k}+\kappa_{k}\left|f_{k}\right|^{2}}\right) d \omega .
$$

Also, substitution of (28) into the constraint (21) yields

$$
\tilde{\boldsymbol{b}}_{d, k} \tilde{\boldsymbol{b}}_{d, k}^{H}=1 .
$$

The optimum TIR for the subchannel $k$ which minimizes (29) subject to the above constraint is the eigenvector corresponding to the smallest eigenvalue of the matrix $\boldsymbol{R}_{k}$.

Remark 8: Note the structural similarity between the channel shortening equalization and the decision feedback equalization (DFE), e.g., [20], [30], and [42]. Under the assumption of correct tentative decisions in the DFE, the structure of both 
equalizers become the same [43]. However, the underlying purpose and design methodology of these equalizers have fundamental differences. Ultimately, the DFE attempts to completely equalize the channel, whereas channel shortening equalizer converts the original channel into a certain partial response channel. The feedforward filter in both cases has the same structure and similar functionalities. However, the feedback filter of the DFE is actually used to cancel the post-cursor ISI, whereas the TIR models the partial response. Moreover, the feedback filter is calculated by Cholesky or spectral factorization, whereas the TIR is found from eigendecomposition. For a detailed discussion on this comparison, the reader is referred to [39].

\section{FREQUENCY-DOMAIN RECEIVER-ONLY DESIGN}

We could set $\boldsymbol{C}(k)=\delta(k) \boldsymbol{I}$, i.e., avoid transmitter preprocessing, and design the receiver filter and the target impulse response accordingly, where $\delta(k)$ is the Kronecker delta function. In this case, only the receiver will be designed using the frequency-domain approach, namely, frequency-domain receiveronly design. The MSE expression in (5) is still valid with the substitution $C(\omega)=I$, however, we can no longer exploit the diagonalization procedure. The receiver filter can be shown to be the same as (9) except $C(\omega)=I$. The TIR can be found by substituting

$$
\begin{aligned}
\boldsymbol{B}(\omega) & =\boldsymbol{B}_{0}+\boldsymbol{B}_{1} e^{-j \omega}+\cdots+\boldsymbol{B}_{n_{b}-1} e^{-j \omega\left(n_{b}-1\right)} \\
& =\left[\begin{array}{lll}
\boldsymbol{B} & \cdots & \boldsymbol{B}_{n_{b}-1}
\end{array}\right]\left[\begin{array}{c}
\boldsymbol{I} \\
\vdots \\
e^{-j \omega\left(n_{b}-1\right)} \boldsymbol{I}
\end{array}\right]=\tilde{\boldsymbol{B}} \boldsymbol{\Omega}
\end{aligned}
$$

into (10) where the matrix taps $\boldsymbol{B}_{i}$ have been defined in (2). Then the MSE expression becomes

$$
J(B)=\boldsymbol{B}^{H} \boldsymbol{R} \boldsymbol{B}
$$

where

$$
\begin{aligned}
& {[\boldsymbol{R}]_{m, n}} \\
& \quad=\frac{1}{2 \pi} \int_{-\pi}^{\pi} e^{-j \omega(m-n)}\left[\boldsymbol{\Phi}_{d d}^{-1}+\boldsymbol{H}^{H}(\omega) \boldsymbol{\Phi}_{n n}^{-1}(\omega) \boldsymbol{H}(\omega)\right]^{-1} d \omega .
\end{aligned}
$$

This matrix can be considered to be in analogy with the matrix $\overline{\boldsymbol{R}}$ in [25] when the length of the FIR filter goes to infinity, i.e., $N_{f} \rightarrow \infty$. Together with the orthogonality constraint, $J(B)$ can be further minimized as explained in Section IV-E.

\section{COMPUTATIONAL COMPLEXITY ANALYSIS}

In this section, we compare the proposed equalizer design with the time-domain MMSE channel shortening design in terms of computational complexity (number of flops) [44], [45]. We define a flop as one of the following operations: addition, subtraction, multiplication, division, or square-root of real scalar floating-point numbers. Contemporary digital signal processor (DSP) integrated circuits (ICs) can perform these operations in a single cycle, e.g., [46]. For complex scalars, addition needs two real summations, i.e., two flops, and multiplication requires four real multiplications and two real summations, i.e., six flops.
The matrix operation used in this analysis, and their complexities can be summarized as follows.

1. Matrix multiplication: For $A \in \mathbb{C}^{m \times n}, \boldsymbol{B} \in \mathbb{C}^{n \times p}, A B$ requires $m p(8 n-2)$ flops for arbitrary matrices or $6 n$ flops for diagonal matrices, respectively.

2. Fast Fourier transform (FFT) and inverse FFT (IFFT) of size $N$ are performed in $5 N \log _{2} N$ flops.

3. Singular value decomposition and eigendecomposition of an $m \times m$ matrix have similar complexities of $126 \mathrm{~m}^{3}$ flops.

4. Inversion of a block-Toeplitz matrix with $n$ diagonal blocks of size of $m \times m$ needs $\mathcal{O}\left(m^{3} n^{2}\right)$ (only multiplications).

5. Inversion of an $m \times m$ positive definite matrix has a complexity of $(4 / 3) m^{3}+12 m^{2}$ flops.

For a fair comparison, we consider the same scenario for both the proposed frequency-domain methods, and the time-domain MMSE design, i.e., there are $n_{T}$ transmit and $n_{R}$ receive antennas, and the corresponding channel has $n_{H}$ taps which is to be shortened to $n_{b}$ taps. Also assume that $n_{i}$ input streams are loaded in both cases.

\section{A. Proposed Method With Joint Transceiver Optimization}

Assuming the frequency range is divided into $N$ bins.

1. The matrices $\boldsymbol{\Phi}_{d d}(\omega), \boldsymbol{H}(\omega)$ and $\boldsymbol{\Phi}_{n n}(\omega)$ are calculated from their time-domain counterparts by performing the FFT which requires a total of $5\left(n_{i}^{2}+n_{T} n_{R}+n_{R}^{2}\right) N \log _{2} N$ flops.

2. For the eigendecomposition of $\boldsymbol{\Phi}_{d d}(\omega)$ and $\boldsymbol{H}^{H}(\omega) \boldsymbol{\Phi}_{n n}^{-1}(\omega) \boldsymbol{H}(\omega), \quad 126 N n_{i}^{3}$ and $126 N n_{T}^{3}$ flops are required, respectively.

3. Calculations of $\boldsymbol{\Phi}_{n n}^{-1}(\omega), \boldsymbol{H}^{H}(\omega) \boldsymbol{\Phi}_{n n}^{-1}(\omega) \boldsymbol{H}(\omega)$ and $\left(\Lambda_{n n}^{-1}(\omega)\right)^{-1}$ require $N\left((4 / 3) n_{R}^{3}+12 n_{R}^{2}\right), N\left(n_{T} n_{R}+\right.$ $\left.n_{T}^{2}\right)\left(8 n_{R}-2\right)$, and $N P$ flops, respectively.

4. Waterfilling: Calculation of $a_{k, l}$ and $b_{k, l} \forall k, l$ requires $6 P N$ flops in total.

The number of iterations needed for finding the waterlevel depends on the channel condition and the SNR. But simulations suggested that at most 100 iterations are adequate to find the solution. For 100 iterations, the waterfilling algorithm requires $4 N P+300$ flops in total.

5. TIR calculations: The integrand for $\left[\boldsymbol{R}_{k}\right]_{m, n}$ can be calculated in $4 N P$ flops, and the inverse Fourier transform integral can be performed using the IFFT with a total of $5 n_{b} N P \log _{2} N$ flops. Using $\left[\boldsymbol{R}_{k}\right]_{m, n}$ the diagonal TIR impulse response vectors $\overline{\boldsymbol{b}}_{d, k}$ are calculated by employing eigendecomposition with $126 P\left(n_{b}\right)^{3}$ flops, followed by FFTs to find $b_{d, k}(\omega)$ using $5 P N \log _{2} N$ flops.

6. We assume that for the transmitter-TIR optimization, three iterations are performed. Therefore, steps 4 and 5 are repeated three times.

7. Using the operations in (14a) and (15), the transmitter, TIR, and receiver filters are calculated in $N\left(n_{T} n_{i}(8 P-2)+\right.$ $\left.P n_{i}\left(8 n_{i}-2\right)\right)$ and

$$
\begin{array}{r}
N\left[10 n_{i}+P+n_{i}\left[P\left(8 n_{i}-2\right)+n_{T}(8 P-2)\right.\right. \\
\left.\left.+n_{R}\left(8 n_{T}-2\right)+n_{R}\left(8 n_{R}-2\right)\right]\right]
\end{array}
$$

flops, respectively. 
8. Finally, the time-domain counterparts of the designed filters are obtained by a series of FFT operations which require $5\left(n_{i} n_{T}+n_{i} n_{R}\right) N \log _{2} N$ flops.

\section{B. Proposed Method With Receiver-Only Optimization}

Assuming the frequency range is divided into $N$ bins.

1. Repeat step 1 of the joint transceiver optimization,

2. The matrix $\left(\boldsymbol{\Phi}_{d d}^{-1}+\boldsymbol{H}^{H} \boldsymbol{\Phi}_{n n}^{-1} \boldsymbol{H}\right)^{-1}$ is calculated using a total of

$$
\begin{aligned}
N\left[8 n_{T}^{3} / 3+24 n_{T}^{2}+\right. & 4 n_{R}^{3} / 3+12 n_{R}^{2} \\
& \left.+n_{T} n_{R}\left(8 n_{R}-2\right)+n_{T}^{2}\left(8 n_{R}-2\right)\right]
\end{aligned}
$$

flops,

3. The matrix $\boldsymbol{R}$ can be calculated from this matrix using $n_{T} n_{b}$ IFFT operations which require $5 n_{T} n_{b} N \log _{2} N$ flops.

4. TIR, $\boldsymbol{B}$, can be calculated using $126\left(n_{T} n_{b}\right)^{3}$ flops.

5. Next the receiver filter $\boldsymbol{Q}_{\text {opt }}(\omega)$ needs

$$
N\left[n_{T}^{2}\left(8 n_{T}-2\right)+n_{T} n_{R}\left(8 n_{T}-2\right)+n_{T} n_{R}\left(8 n_{R}-2\right)\right]
$$

flops.

6. Finally, to find the time-domain filter coefficients $n_{T} n_{R}$ IFFTs require $5 n_{T} n_{R} N \log _{2} N$ flops.

\section{Time-Domain MMSE Equalizer}

For this analysis, we will follow the derivations in [25] with a slight change in notation. We will use the following substitutions: $n_{q}=N_{f}, n_{b}=N_{b}+1$, and $n_{H}=\nu+1$.

1. Calculation of $\boldsymbol{R}_{y y}$ requires a total of

$$
\begin{aligned}
n_{q} n_{R}\left(8 n_{T}\left(n_{H}+n_{q}-1\right)-2\right) \\
\left(n_{q} n_{R}+n_{T}\left(n_{H}+n_{q}-1\right)\right)+2\left(n_{q} n_{R}\right)^{2}
\end{aligned}
$$

flops. Besides, the inverse $\boldsymbol{R}_{y y}^{-1}$ needs $\mathcal{O}\left(n_{R}^{3} n_{q}^{2}\right)$ complex multiplications.

2. To find $R^{\perp}$ we need a total of

$$
\begin{aligned}
& n_{T}\left(n_{H}+n_{q}-1\right)\left[n_{R} n_{q}\left(8 n_{T}\left(n_{H}+n_{q}-1\right)-2\right)\right. \\
& \quad+n_{R} n_{q}\left(8 n_{R} n_{q}-2\right)+n_{T}\left(n_{H}+n_{q}-1\right)\left(8 n_{R} n_{q}-2\right) \\
& \left.\quad+2 n_{T}\left(n_{H}+n_{q}-1\right)\right]
\end{aligned}
$$

flops.

3. To find the minimum MSE and the corresponding TIR, $\boldsymbol{B}_{\text {opt }}$, the eigendecomposition of a window of $\boldsymbol{R}^{\perp}$, i.e., $\overline{\boldsymbol{R}}$, has to be taken $\left(n_{H}+n_{q}-n_{b}\right)$ times, yielding a complexity of $126\left(n_{H}+n_{q}-n_{b}\right)\left(n_{T} n_{b}\right)^{3}$ flops.

4. Finally, the receiver filter $Q$ can be found in $n_{T} n_{q} n_{R}\left(8 n_{T} n_{b}-2\right)$ flops.

The complexity of the proposed frequency-domain methods is a function of the FFT size $N$, whereas the complexity of the time-domain equalizer mainly depends on the filter length $n_{q}$. The results for various $N$ and $n_{q}$ values are given in Table I for a scenario where an $n_{R}=2 \times n_{T}=2$ MIMO channel of length $n_{H}=10$ is shortened to length $n_{b}=2$ when all
TABLE I

COMPARISON OF THE COMPUTATIONAL COMPLEXITY OF THE PROPOSED JOINT TRANSCEIVER METHOD AND THE TIME-DOMAIN MMSE METHOD IN TERMS OF FLOPS. AN $n_{R}=2 \times n_{T}=2$ MIMO CHANNEL OF LENGTH $n_{H}=10$ IS SHORTENED TO LENGTH $n_{b}=2$

\begin{tabular}{|r|r|r|r|r|}
\hline \multicolumn{2}{|c|}{ Frequency-Domain } & \multicolumn{2}{l|}{ Time-Domain } \\
\hline & \multicolumn{2}{|c|}{$\begin{array}{c}\text { Joint } \\
\text { Transceiver }\end{array}$} & $\begin{array}{c}\text { Receiver } \\
\text { Only }\end{array}$ & \multicolumn{2}{|c|}{} \\
\hline$N$ & \multicolumn{2}{|c|}{ Computational } & $n_{q}$ & $\begin{array}{c}\text { Computational } \\
\text { Complexity }\end{array}$ \\
\hline 64 & 245,000 & 76,000 & 8 & 710,000 \\
128 & 511,000 & 156,000 & 16 & $2,927,000$ \\
256 & $1,070,000$ & 330,000 & 32 & $16,005,000$ \\
\hline
\end{tabular}

the possible eigenmodes are loaded. We observe that the joint transceiver with FFT size $N=256$ has similar computational complexity to the time-domain equalizer with $n_{q}=8$ taps, whereas the computation of the frequency-domain receiver-only equalizer requires even lower complexity compared to both the joint-transceiver and time-domain approaches. From Table I, we observe that the computational complexity of the frequency-domain approach increases linearly with the FFT size, however, the processing requirements of the time-domain approach increase exponentially with the filter length.

Remark 9: An important issue arises in the selection of the FFT size. The proposed method in fact considers continuous frequency which may have an infinite length impulse response. However, for implementation purposes, one needs to perform sampling in the frequency domain. If the sampling period, i.e., the FFT size, is shorter than the effective length of the filter impulse response, aliasing will occur in the time domain which will destroy the equalizer characteristics. Therefore, the FFT size should be chosen long enough to cover the effective equalizer impulse response. In simulations, we observed that an FFT size of $N=256$ is adequate.

Remark 10: In the above computational complexity analysis, we determined the complexity of computing the required parameters for the proposed frequency-domain approach and the time-domain channel shortening approach. Once these parameters are determined, both the transmitter and the receiver can directly be implemented using $N$-tap FIR filters with matrix coefficients of dimensions $n_{T} \times n_{i}$ and $n_{i} \times n_{R}$, respectively. Similarly, the implementation of the time-domain equalizer can be realized as a single $n_{q}$-tap FIR filter with matrix coefficients of dimensions $n_{i} \times n_{R}$. In the simulations section, it will be demonstrated that the performance of the time-domain approach comes closer to our proposed frequency-domain approach (receiveronly design) only when the filter length $n_{q}$ is very high. However, the computational cost of determining the optimum filter parameters according to the time-domain approach is significantly higher than that of the frequency-domain approach. Therefore, in systems where the complexity of determining the receiver parameters dominates the implementation complexity, the frequency-domain approach could be proven to be advantageous.

Remark 11: If the implementation complexity, i.e., filter length in the transceiver, is also an important issue besides the computational complexity associated with designing the filters, 


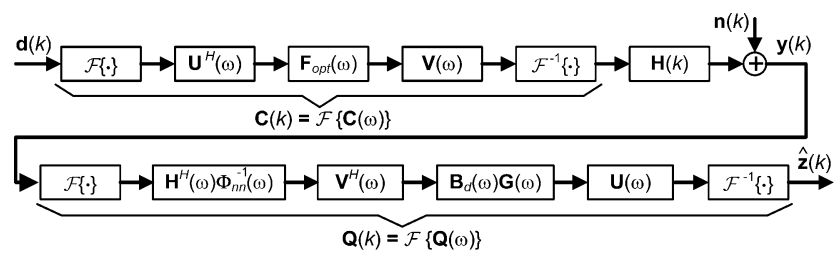

Fig. 4. Implementation of broadband MIMO beamformer.

one may opt for computing a shorter version of the proposed equalizer. There exist several methods for this purpose. The first one is to approximate the long impulse response of the calculated filter by an IIR filter. However, care should be taken to ensure stability, since the proposed method does not explicitly impose any conditions on the stability of such an IIR filter. One method for designing such a stable IIR filter is proposed in, e.g., [47]. Another method is to employ a model reduction technique, which may introduce significant complexity while reducing the finite impulse response (FIR) filter size. A third approach is to exploit the degrees of freedom associated with the phase selection at the transmitter and the receiver to reduce the impulse response length [41].

\section{BROADBAND MIMO BEAMFORMER}

A demonstration of the proposed frequency-domain-based technique is depicted in Fig. 4 where $\mathcal{F}\{\cdot\}$ and $\mathcal{F}^{-1}\{\cdot\}$ represent, respectively, the Fourier and inverse Fourier transforms. Although the transmitter and receiver filters are physically implemented in the time domain, i.e., as $\boldsymbol{C}(k)$ and $\boldsymbol{Q}(k)$, for the purpose of analysis, we consider their frequency-domain counterparts. The block $\boldsymbol{U}(\omega)$ removes the spatial correlation of the input $\boldsymbol{d}(k)$ in the frequency domain. Likewise, the matrix $\boldsymbol{V}(\omega)$ obtained from the singular value decomposition of the matrix $\boldsymbol{H}^{H}(\omega) \Phi_{n n}^{-1}(\omega) \boldsymbol{H}(\omega)$ at each frequency $\omega$ is used to steer MIMO beams along each spatial-frequency eigenmode. The diagonal transmitter operator $\boldsymbol{F}_{\text {opt }}(\omega)$ performs spatial and frequency waterfilling as explained before under the constraints on the length of the overall impulse response and the total transmit power. The diagonal filter $\boldsymbol{B}_{d}(\omega) \boldsymbol{G}(\omega)$, jointly with $\boldsymbol{F}_{\text {opt }}(\omega)$, performs channel shortening equalization at each eigenmode so that the length of the impulse response of the equalized channel (i.e., the combination of the transmitter filter, the actual channel and the receiver filter) does not exceed a specified value. When the number of input streams $n_{i}$ is one, $\boldsymbol{V}(\omega)$ will be the dominant left singular value of $\boldsymbol{H}(\omega)$ at each frequency $\omega$, resulting in a coherent transmitter (beamformer) which directs the total transmit power into the dominant eigenmodes at each frequency. However, the overall framework will ensure that the effective channel impulse response in the time domain is shortened to a specified value.

Equalization of the remaining paths (i.e., after channel shortening) can be performed using a Viterbi equalizer. Compared to a complete equalization (i.e., equalized to a single spike), MLSE combined with channel shortening provides extra degrees of freedom for equalization and noise suppression. This broadband beamformer differs from the broadband beamformer proposed in [30] as our approach imposes a constraint on the length of the effective channel. Hence, this treats a very general framework, and the case for complete equalization can be obtained by setting the TIR length to one.

\section{Simulations AND Results}

There are two measures of performance adopted in this study. The first one is the shortening signal-to-interference-plus-noise ratio (SINR) at the output of the channel shortening filter. It is defined as

$$
\mathrm{SINR}=\frac{E_{\text {win }}}{E_{\text {wall }}+E_{\text {noise }}}
$$

where $E_{\text {win }}$ is the energy of the signal within the time window defined by the finite-length TIR, $E_{\text {wall }}$ is the energy of the signal outside this window, and $E_{\text {noise }}$ is the total noise energy. The second measure is the bit-error-rate (BER) performance. Note that the SINR metric is similar to the shortening SNR (SSNR) metric in [21] with the addition of the noise power term $E_{\text {noise }}$.

The performance of the proposed frequency-domain approach for both the receiver-only and the joint transceiver based schemes is compared with that of the finite-length time-domain MMSE scheme of [25]. A randomly generated $n_{H}=10$ tap $\left(n_{R}=2\right) \times\left(n_{T}=2\right)$ MIMO channel is shortened to an $n_{b}=2$ tap $2 \times 2$ MIMO channel. The channel taps are assumed to be zero-mean circularly symmetric, white complex Gaussian random variables, i.e., they are both spatially and temporally uncorrelated, and they are normalized so that the variance of the channel is unity. Even though a more realistic scenario would scale the taps according to a certain power delay profile, we have chosen this setup to account for a bad channel. The noise vector $\boldsymbol{n}(k)$ is composed of zero-mean circularly symmetric, white complex Gaussian random variables. For all the simulations we use quaternary phase-shift keying (QPSK) modulation except the last one. For the frequency-domain design the FFT size is chosen as $N=256$ whereas for the time-domain design, various filter lengths $n_{q}=16,32,64$, and 256 are considered. As discussed in Section VI, the computational complexity of $N=256$ and $n_{q}=8$ are comparable, and as $n_{q}$ increases, the computational complexity of the time-domain design increases exponentially. However, the performance of the time-domain equalizer with $n_{q}=8$ is very poor. Therefore, instead, we will consider the case $n_{q}=16$ as the lowest value of $n_{q}$. For the joint transceiver design, the number of iterations used for the transmitter filter and TIR optimization is ten, however, it was observed three or four iterations were adequate most of the time.

Fig. 5 provides the SINR performance comparison of the proposed methods and the time-domain design. It is seen that the joint transceiver design outperforms all other approaches in terms of SINR. Even with longer filter lengths, i.e., $n_{q}=256$, the time-domain design under-performs both the frequency-domain joint-transceiver and receiver-only equalizers. One interesting observation about the time-domain FIR filter is the appearance of error floors. This is because a short FIR filter does not have enough degrees of freedom to completely equalize a FIR channel and there will be significant residual error yielding a loss in performance. However, as the filter length increases its performance gets closer to that of the frequency-domain receiver-only design. Therefore, this set of simulations also 


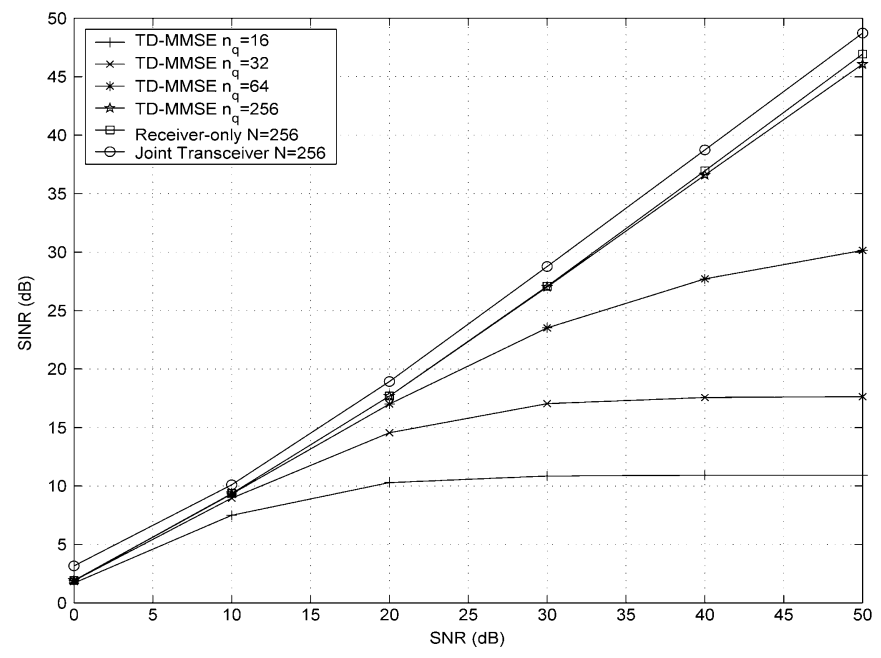

Fig. 5. SINR comparison of the finite-length time-domain design for filter lengths, $n_{q}=16,32,64$, and 256 to the proposed frequency-domain designs with both joint transceiver and receiver only processing $(N=256)$ for an $n_{T}=2$ transmit, $n_{R}=2$ receive antenna scenario where the channel of length $n_{H}=10$ is shortened to $n_{b}=2$ taps.

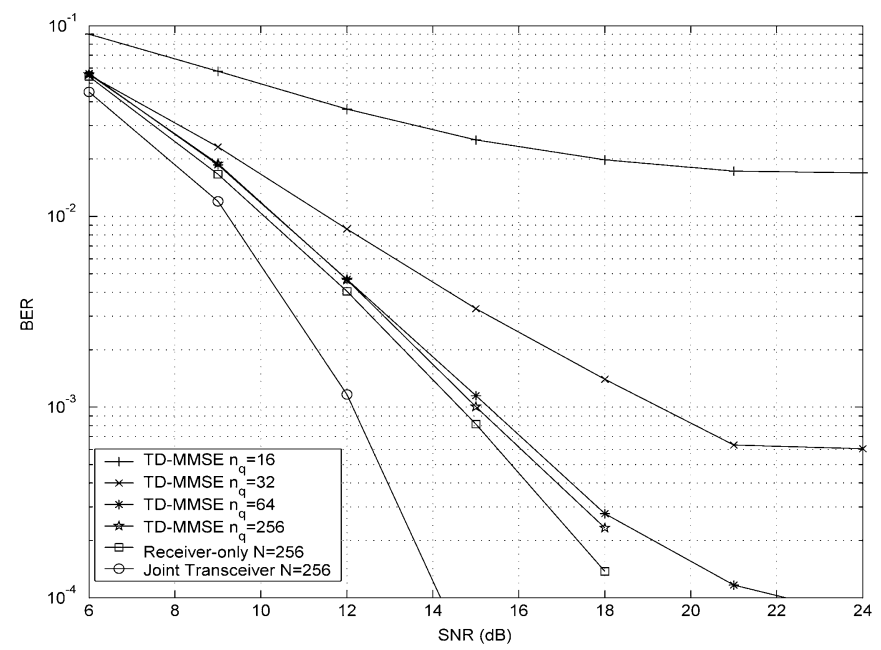

Fig. 6. BER comparison of the finite-length time-domain design for filter lengths, $n_{q}=16,32,64$, and 256 to the proposed frequency-domain designs with both joint transceiver and receiver only processing $(N=256) . n_{T}=2$, $n_{R}=2$, and the channel of length $n_{H}=10$ is shortened to $n_{b}=2$ taps.

demonstrates the equivalence of the infinite-length time-domain approach and the frequency-domain receiver-only approach. Hence, the frequency-domain receiver-only design always outperforms the FIR design. Moreover, due to the additional degrees of freedom at the transmitter, the performance of the joint transceiver design is superior to both of them with a gain of $3 \mathrm{~dB}$ over the frequency-domain receiver-only equalizer.

In Fig. 6, we examine the BER performance of the proposed frequency-domain joint transceiver and receiver-only methods and compare them to the BER performance of the time-domain channel shortening equalizer. Both of the two eigenmodes arising from this scenario are loaded $\left(n_{i}=2\right)$. In order to detect the transmitted data in this spatial multiplexing scenario, an MLSE receiver is employed at the receiver end.

Note that the front-end channel shortening equalizer makes the noise colored, and this coloring should be incorporated into

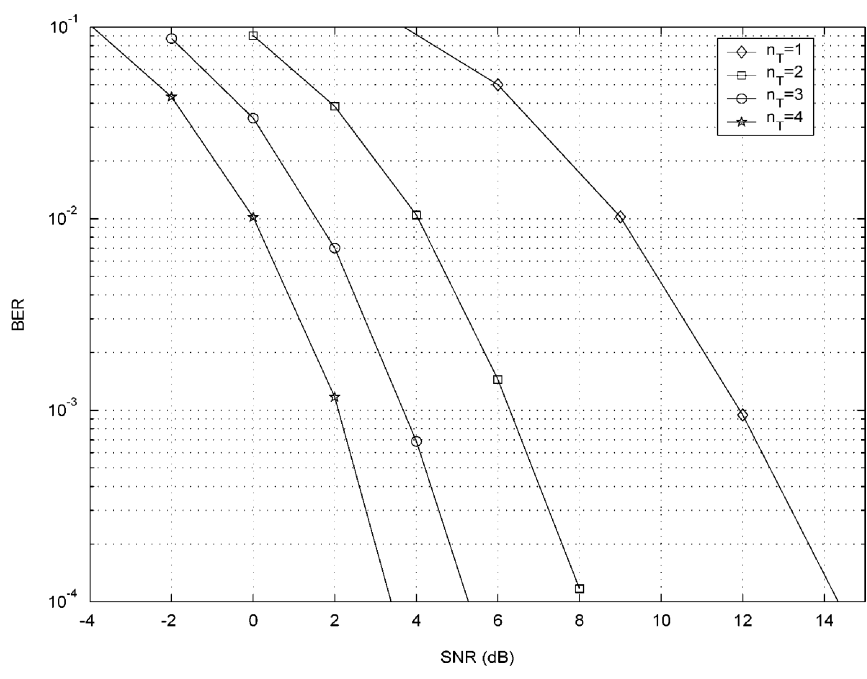

Fig. 7. Demonstration of the BER performance of the proposed joint transceiver design when the number of receive antennas is fixed to one and the number of transmit antennas varies between one and four.

the MLSE algorithm for optimum operation. However, this increases the computational complexity of the algorithm, which is in contrast to the purpose of channel shortening. A suboptimum, but with lower complexity MLSE algorithm would assume uncolored noise.

It has been demonstrated in [26] that incorporating only the zeroth lag of the covariance of the noise into the MLSE algorithm does not significantly increase the complexity, but enhances the performance. Hence, we treat the noise in the MLSE algorithm as temporally white, but incorporate the spatial correlation as in [26].

From the BER curves in Fig. 6, we observe that even though the proposed frequency-domain method has a relatively lower computational complexity, it significantly outperforms the time-domain equalizer for all $n_{q}$ values. The performance of the time-domain design approaches that of the receiver-only frequency-domain design only for very long filter lengths. For shorter filter lengths, e.g., $n_{q}=16$ and 32 , the time-domain equalizer suffers from a severe error-floor effect. We also observe that the transmitter processing provides a significant gain over the receiver- only equalization, e.g., at $\mathrm{BER}=10^{-3}, 2.5-\mathrm{dB}$ gain is observed. We also note the diversity gain obtained using transmitter processing.

Fig. 7 provides the simulation results when the number of transmit antennas varies from one to four but the receiver has a single antenna. The multiple-input single-output (MISO) channel of length $n_{H}=10$ is shortened to $n_{b}=2$ using the proposed joint transceiver design. Apparently, there is only a single spatial eigenmode, hence, the number of input streams is fixed to one. As it can be observed from Fig. 7, there are significant diversity and array gains that shift the curve to the left as the number of transmit antennas increases.

Finally, in Fig. 8, we demonstrate that the proposed technique can be considered as a broadband beamformer with constraints on the multipath length. A $2 \times 2 \mathrm{MIMO}$ channel with $n_{H}=10$ multipath components is shortened to a length $n_{b}=22 \times 2$ MIMO channel. A spatial-multiplexing scheme employing an 


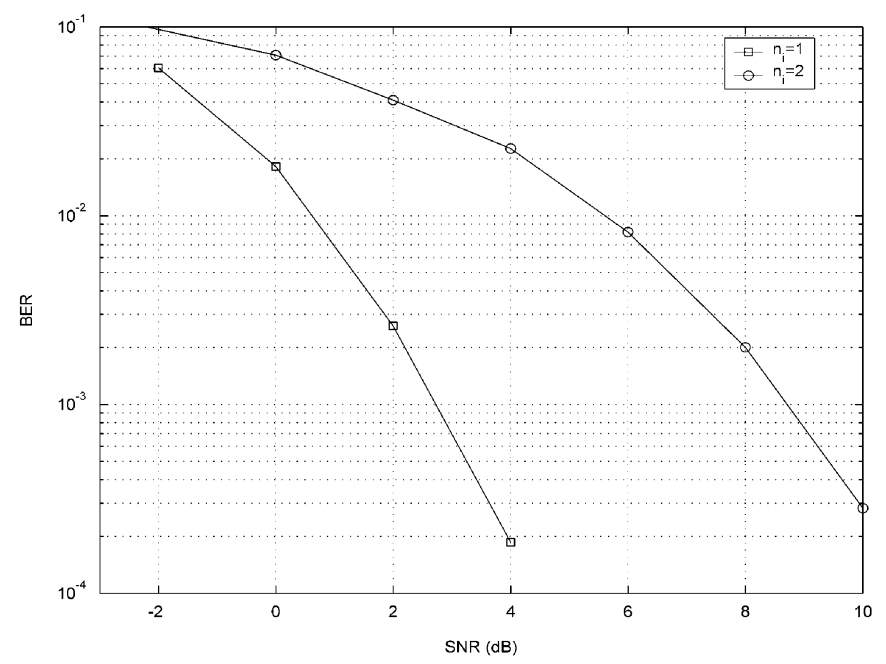

Fig. 8. Demonstration of diversity gain corresponding to the proposed joint transceiver structure when $n_{i}=1$ and 2 for the $2 \times 2$ MIMO channel.

MLSE receiver (to process the remaining two paths) is considered. Two scenarios are examined. In the first one, only one of the two eigenmodes with the lowest MSE is selected for transmission resulting in a beamformer in each frequency bin. In the second scenario, both eigenmodes are loaded with data streams, and the transmit power is distributed among these channels according to the proposed space-frequency waterfilling algorithm. In order to keep the throughput unchanged for both scenarios, we used QPSK modulation for $n_{i}=1$ and binary phase-shift keying (BPSK) for $n_{i}=2$. From the slopes of the curves it can be observed that loading the best out of two subchannels (i.e., $n_{i}=1$ ) provides twice the order of diversity as compared to when both eigenmodes are activated $\left(n_{i}=2\right)$.

When both modes are used, the diversity order is approximately two as the effective length of the shortened channel used for MLSE is two. However, when $n_{i}=1$ (i.e., only the strongest subchannel is used), the diversity order increases significantly (greater than four). This is due to the diversity introduced by the transmitter and the receiver antennas in addition to the multipaths. Hence, the proposed technique provides a tradeoff between the data rate (multiplexing), the diversity gain, and the complexity of the MLSE receiver characterized by the length of the resulting shortened channel.

\section{CONCLUSION}

A frequency-domain approach which includes both the receiver-only and the joint transceiver design was proposed for MIMO channel shortening under the MMSE criterion. It was demonstrated that the proposed frequency-domain receiver-only equalizer is effectively equivalent to an infinitelength time-domain equalizer. For the joint transceiver design, it was proven that under certain transformations and conditions, the problem could be cast into transmission over parallel independent SISO subsystems, significantly simplifying the design. A significant gain in terms of SINR ratio and BER was observed when the transmitter preprocessor was employed. A practical space-frequency waterfilling algorithm was also provided for the transmitter filter design. The computational complexity of determining the filters using the frequency-domain approach is considerably lower than that of the time-domain approach. Finally, we demonstrated that our proposed method could also be considered as a broadband MIMO beamformer with constraints on the time-domain multipath length, hence it provides a tradeoff among the data rate (spatial multiplexing), the diversity gain, and the complexity of the MLSE.

\section{APPENDIX I \\ PROOF OF THEOREM 1}

We start the proof by first deriving two key equations that will be used in the calculations of several matrices in the following sections. Later, Theorem 1 will be proven on the bases of these structures.

Substituting the definitions in (14) and (15) and the decompositions in (11) and (12) into (5), the MSE becomes

$$
\begin{array}{r}
J\left(\boldsymbol{Q}, \boldsymbol{F}, \boldsymbol{B}_{d}\right)=\frac{1}{2 \pi} \operatorname{tr} \int_{-\pi}^{\pi} \boldsymbol{B}_{d}\left\{[\boldsymbol{I}-\boldsymbol{W} \boldsymbol{F}] \boldsymbol{K}_{d d}[\boldsymbol{I}-\boldsymbol{W} \boldsymbol{F}]\right. \\
\left.+\boldsymbol{W} \boldsymbol{\Lambda}_{n n} \boldsymbol{W}^{H}\right\} \boldsymbol{B}_{d}^{H} d \omega
\end{array}
$$

where $\boldsymbol{W}=\boldsymbol{G} \boldsymbol{\Lambda}_{n n}^{-1}$ and we used the property $\operatorname{tr}\{\boldsymbol{A} \boldsymbol{B}\}=$ $\operatorname{tr}\{\boldsymbol{B} \boldsymbol{A}\}$ for appropriate dimensions. By setting the derivative of (33) with respect to $\boldsymbol{W}^{H}$ to zero, we obtain

$$
\begin{aligned}
\boldsymbol{K}_{d d} \boldsymbol{F}^{H} & =W\left(\boldsymbol{F} \boldsymbol{K}_{d d} \boldsymbol{F}^{H}+\boldsymbol{\Lambda}_{n n}\right) \\
\boldsymbol{W} & =\boldsymbol{K}_{d d} \boldsymbol{F}^{H}\left(\boldsymbol{F} \boldsymbol{K}_{d d} \boldsymbol{F}^{H}+\boldsymbol{\Lambda}_{n n}\right)^{-1} .
\end{aligned}
$$

Also, constructing the Lagrangian using (33) and (20b) with a Lagrange multiplier $\gamma$, and equating the differentiation with respect to $\boldsymbol{F}$ to zero, we obtain

$$
\boldsymbol{B}_{d}^{H} \boldsymbol{B}_{d} \boldsymbol{W}=\boldsymbol{F}^{H} \boldsymbol{W}^{H} \boldsymbol{B}_{d}^{H} \boldsymbol{B}_{d} \boldsymbol{W}+\gamma \boldsymbol{F}^{H} .
$$

Proposition 1: $\boldsymbol{W F}$ is diagonal.

Proof: Postmultiplying (35) with $\boldsymbol{F}$ we obtain

$$
\boldsymbol{B}_{d}^{H} \boldsymbol{B}_{d} \boldsymbol{W} \boldsymbol{F}=\boldsymbol{F}^{H} \boldsymbol{W}^{H} \boldsymbol{B}_{d}^{H} \boldsymbol{B}_{d} \boldsymbol{W} \boldsymbol{F}+\gamma \boldsymbol{F}^{H} \boldsymbol{F} .
$$

Since the right-hand side is Hermitian symmetric, the left-hand side must also be Hermitian symmetric. Substituting (34b) results in

$$
\begin{aligned}
\boldsymbol{B}_{d}^{H} \boldsymbol{B}_{d} \boldsymbol{W} \boldsymbol{F} & =\boldsymbol{B}_{d}^{H} \boldsymbol{B}_{d} \boldsymbol{K}_{d d} \boldsymbol{F}^{H}\left(\boldsymbol{F} \boldsymbol{K}_{d d} \boldsymbol{F}^{H}+\boldsymbol{\Lambda}_{n n}\right)^{-1} \boldsymbol{F} \\
& =\boldsymbol{\Sigma} \boldsymbol{K}_{d d} \boldsymbol{\Upsilon}
\end{aligned}
$$

where $\boldsymbol{\Sigma}=B_{d}^{H} \boldsymbol{B}_{d}$ and $\boldsymbol{\Upsilon}=F^{H}\left(\boldsymbol{F} \boldsymbol{K}_{d d} \boldsymbol{F}^{H}+\boldsymbol{\Lambda}_{n n}\right)^{-1} \boldsymbol{F}$ are again Hermitian symmetric and $\boldsymbol{K}_{d d}$ is diagonal by definition. Since for a Hermitian symmetric matrix $A=A^{H}$, (36) can be written as $\boldsymbol{B}_{d}^{H} \boldsymbol{B}_{d} \boldsymbol{W} \boldsymbol{F}=\left(\boldsymbol{B}_{d}^{H} \boldsymbol{B}_{d} \boldsymbol{W} \boldsymbol{F}\right)^{H}$, hence

$$
\boldsymbol{\Sigma} \boldsymbol{K}_{d d} \boldsymbol{\Upsilon}=\left(\boldsymbol{\Sigma} \boldsymbol{K}_{d d} \boldsymbol{\Upsilon}\right)^{H}=\boldsymbol{\Upsilon}^{H} \boldsymbol{K}_{d d} \boldsymbol{\Sigma}^{H}=\boldsymbol{\Upsilon} \boldsymbol{K}_{d d} \boldsymbol{\Sigma} .
$$

Therefore, we have

$$
\Sigma K_{d d} \Upsilon=\Upsilon \boldsymbol{K}_{d d} \boldsymbol{\Sigma}
$$

which is possible only when $\boldsymbol{\Sigma}=\boldsymbol{\Upsilon}$ or $\boldsymbol{\Sigma}$ and $\boldsymbol{\Upsilon}$ are both diagonal. Inspection of (36) reveals that $\Sigma \neq \Upsilon$. Therefore, we conclude that $\boldsymbol{\Sigma}=\boldsymbol{B}_{d}^{H} \boldsymbol{B}_{d}$ and $\boldsymbol{\Upsilon}=\boldsymbol{F}^{H}\left(\boldsymbol{F} \boldsymbol{K}_{d d} \boldsymbol{F}^{H}+\boldsymbol{\Lambda}_{n n}\right)^{-1} \boldsymbol{F}$ are diagonal. Hence, $\boldsymbol{W} \boldsymbol{F}=\boldsymbol{K}_{d d} \Upsilon$ is diagonal. 
Using the matrix inversion lemma, we can rewrite the matrix $\left[\boldsymbol{K}_{d d}^{-1}+\boldsymbol{F}^{H} \boldsymbol{\Lambda}_{n n}^{-1} \boldsymbol{F}\right]^{-1}$ as

$$
\begin{aligned}
& {\left[\boldsymbol{K}_{d d}^{-1}+\boldsymbol{F}^{H} \boldsymbol{\Lambda}_{n n}^{-1} \boldsymbol{F}\right]^{-1}} \\
& \quad=\boldsymbol{K}_{d d}-\boldsymbol{K}_{d d} \boldsymbol{F}^{H}\left(\boldsymbol{F} \boldsymbol{K}_{d d} \boldsymbol{F}^{H}+\boldsymbol{\Lambda}_{n n}\right)^{-1} \boldsymbol{F} \boldsymbol{K}_{d d} .
\end{aligned}
$$

The second term in the right-hand side is written as $\boldsymbol{W} \boldsymbol{F} \boldsymbol{K}_{d d}=\boldsymbol{K}_{d d} \boldsymbol{F}^{H}\left(\boldsymbol{F} \boldsymbol{K}_{d d} \boldsymbol{F}^{H}+\boldsymbol{\Lambda}_{n n}\right)^{-1} \boldsymbol{F} \boldsymbol{K}_{d d}$. However, $\boldsymbol{W} \boldsymbol{F}$ has been proven to be diagonal. Hence, $\boldsymbol{W} \boldsymbol{F} \boldsymbol{K}_{d d}$ is diagonal, and so is $\left[\boldsymbol{K}_{d d}^{-1}+\boldsymbol{F}^{H} \boldsymbol{\Lambda}_{n n}^{-1} \boldsymbol{F}\right]^{-1}$, completing the proof of Theorem 1.

\section{APPENDIX II}

PROOF OF THEOREM 2

Write the arbitrary matrix $\boldsymbol{B}_{d}(\omega)$ in a vector form as follows:

$$
\begin{aligned}
\boldsymbol{B}_{d}(\omega) & =\boldsymbol{B}_{d, 0}+\cdots+\boldsymbol{B}_{d, n_{b}} e^{-j \omega\left(n_{b}-1\right)} \\
& =\left[\begin{array}{lll}
\boldsymbol{B}_{d, 0}^{T} & \cdots & \boldsymbol{B}_{d,\left(n_{b}-1\right)}^{T}
\end{array}\right]\left[\begin{array}{c}
\boldsymbol{I}_{n_{i}} \\
\vdots \\
e^{-j \omega\left(n_{b}-1\right)} \boldsymbol{I}_{n i}
\end{array}\right] .
\end{aligned}
$$

Define $\tilde{\boldsymbol{b}}_{d}^{T} \triangleq\left[\begin{array}{lll}\boldsymbol{B}_{d, 0}^{T} & \cdots & \boldsymbol{B}_{d,\left(n_{b}-1\right)}^{T}\end{array}\right]$. Using (31) in (19) we can write the MSE as

$$
J=\operatorname{tr} \tilde{\boldsymbol{B}}_{d}^{T} \boldsymbol{R} \tilde{\boldsymbol{B}}_{d}^{*}
$$

where the $n_{i} n_{b} \times n_{i} n_{b}$ matrix $\boldsymbol{R}$ is

$$
\begin{aligned}
& \boldsymbol{R}=\frac{1}{2 \pi} \int_{-\pi}^{\pi}\left[\begin{array}{c}
1 \boldsymbol{I} \\
\vdots \\
e^{-j \omega\left(n_{b}-1\right)} \boldsymbol{I}
\end{array}\right]\left[\boldsymbol{K}_{d d}^{-1}+\boldsymbol{F}^{H} \boldsymbol{\Lambda}_{n n}^{-1} \boldsymbol{F}\right]^{-1} \\
& {\left[\begin{array}{lll}
1 \boldsymbol{I} & \cdots & e^{j \omega\left(n_{b}-1\right)} \boldsymbol{I}
\end{array}\right] d \omega .}
\end{aligned}
$$

Note that the term $\left[\boldsymbol{K}_{d d}^{-1}(\omega)+\boldsymbol{F}^{H}(\omega) \boldsymbol{\Lambda}_{n n}^{-1}(\omega) \boldsymbol{F}(\omega)\right]^{-1}$ is diagonal, and the integral in (39) is the inverse Fourier transform converting the spectral density function $\left[\boldsymbol{K}_{d d}^{-1}+\boldsymbol{F}^{H} \boldsymbol{\Lambda}_{n n}^{-1} \boldsymbol{F}\right]^{-1}$ into a correlation matrix $\boldsymbol{R}$ working in a block-by-block basis. It can be shown that $\boldsymbol{R}$ is a block Toeplitz matrix where each block is a diagonal matrix, i.e.,

$$
\boldsymbol{R}=\left[\begin{array}{ccccc}
\boldsymbol{\Delta}_{0} & \boldsymbol{\Delta}_{1} & \boldsymbol{\Delta}_{2} & \cdots & \boldsymbol{\Delta}_{n_{b}-1} \\
\boldsymbol{\Delta}_{1}^{H} & \boldsymbol{\Delta}_{0} & \boldsymbol{\Delta}_{1} & & \boldsymbol{\Delta}_{n_{b}-2} \\
\boldsymbol{\Delta}_{2}^{H} & \boldsymbol{\Delta}_{1}^{H} & \boldsymbol{\Delta}_{0} & & \vdots \\
\vdots & & & \ddots & \vdots \\
\boldsymbol{\Delta}_{n_{b}-1}^{H} & \boldsymbol{\Delta}_{n_{b}-2}^{H} & \cdots & \cdots & \boldsymbol{\Delta}_{0}
\end{array}\right]
$$

where

$$
\boldsymbol{\Delta}_{m}=\left[\begin{array}{cccc}
\delta_{m, 0} & 0 & \cdots & 0 \\
0 & \delta_{m, 1} & & 0 \\
\vdots & & \ddots & \vdots \\
0 & 0 & & \delta_{m, n_{i}-1}
\end{array}\right],
$$

for some scalar $\delta_{m, n}$ determined by the numerical evaluation of (39).

As stated in Section IV-E, the coefficients of the TIR are assigned as the eigenvectors of $\boldsymbol{R}$. Using the relation $(\boldsymbol{R}-\lambda \boldsymbol{I}) \boldsymbol{b}=$ $\mathbf{0}$, where $\lambda$ is one of the $n_{i} n_{b}$ eigenvalues of $\boldsymbol{R}$ and $\boldsymbol{b}$ is the corresponding $n_{i} n_{b} \times 1$ eigenvector, it can be shown that $\boldsymbol{b}$ should be in the following form when the structure of $\boldsymbol{R}$ is as in (40) and (41):

$\boldsymbol{b}=\left[\mathbf{0}_{1 \times m} \tilde{b}_{k, 0} \mathbf{0}_{1 \times n_{i}-m-1}\left|\mathbf{0}_{1 \times m} \tilde{b}_{k, 1} \mathbf{0}_{1 \times n_{i}-m-1}\right|\right.$

$$
\left.\cdots \mid \mathbf{0}_{1 \times m} \tilde{b}_{k, n_{b}-1} \mathbf{0}_{1 \times n_{i}-m-1}\right]^{T}
$$

where $\tilde{b}_{k, l}, l=0, \ldots, n_{b}-1$, are some possibly nonzero, complex-valued scalars. For the first set of $n_{b}$ eigenvectors corresponding to the first entries of the matrices $\Delta_{m}, l$ is equal to 0 . Similarly, there are $n_{b}$ eigenvectors corresponding to the second entries of the matrices $\Delta_{m}$, with $l=1$, and so on, for all $l$ yielding a total of $n_{i} n_{b}$ eigenvectors. For more details see [39].

Recall that there are $n_{i}$ subchannels corresponding to the $n_{i}$ input data streams. Since each diagonal entry of (41) corresponds to a separate subchannel, the scalars $\left\{\tilde{b}_{k, l}\right\}$ forming the "diagonal" target impulse for the $k$ th subchannel are found as the elements of the eigenvector corresponding to the smallest eigenvalue of the following "sub"-correlation matrix constructed from $\boldsymbol{R}$ by taking the $k$ th diagonal entry of (41)

$$
\boldsymbol{R}_{k}=\left[\begin{array}{cccc}
\delta_{0, k} & \delta_{1, k} & \cdots & \delta_{n b, k} \\
\delta_{1, k}^{*} & \delta_{0, k} & & \delta_{n b-1, k} \\
\vdots & & \ddots & \vdots \\
\delta_{n b, k}^{*} & \delta_{n b-1, k}^{*} & \cdots & \delta_{0, k}
\end{array}\right] .
$$

These $n_{i}$ eigenvectors, $\tilde{\boldsymbol{b}}_{\text {opt }, k}$, from (42), determined as explained above, constitute the columns of the optimum $\tilde{\boldsymbol{b}}_{d}$ matrix in (38), i.e., as shown at the bottom of the page. Equivalently, substituting $\tilde{\boldsymbol{B}}_{d, \mathrm{opt}}$ into (31) we have

$$
\begin{gathered}
\boldsymbol{B}_{d, \mathrm{opt}}(\omega) \\
=\left[\begin{array}{cccc}
\tilde{b}_{0,0} & 0 & \cdots & 0 \\
0 & \tilde{b}_{1,0} & & 0 \\
\vdots & & \ddots & \vdots \\
0 & 0 & \cdots & \tilde{b}_{n_{i}, 0}
\end{array}\right]+\cdots
\end{gathered}
$$

$$
\begin{aligned}
& \tilde{\boldsymbol{B}}_{d, \mathrm{opt}}=\left[\begin{array}{lll}
\tilde{\boldsymbol{b}}_{\mathrm{opt}, 0} & \cdots & \tilde{\boldsymbol{b}}_{\mathrm{opt}, n_{i}}
\end{array}\right] \\
& =\left[\begin{array}{cccc|c|cccc}
\tilde{b}_{0,0} & 0 & \cdots & 0 & \cdots & \tilde{b}_{0,\left(n_{b}-1\right)} & 0 & \cdots & 0 \\
0 & \tilde{b}_{1,0} & & 0 & & \tilde{b}_{1,\left(n_{b}-1\right)} & & 0 \\
\vdots & & \ddots & \vdots & & \vdots & & \ddots & \vdots \\
0 & 0 & \cdots & \tilde{b}_{n_{i}, 0} & \cdots & 0 & 0 & \cdots & \tilde{b}_{n_{i},\left(n_{b}-1\right)}
\end{array}\right]^{T} .
\end{aligned}
$$






Hence $\boldsymbol{B}_{d, \mathrm{opt}}(\omega)$ is diagonal.

\section{APPENDIX III \\ PROOF OF THEOREM 3}

The proof is composed of three parts. First $\boldsymbol{F}$ and $\boldsymbol{W}^{H}$ will be shown to have $P-n_{i}$ all zero rows, in part i). Using this property they will be reduced to full rank square matrices of dimension $n_{i} \times n_{i}$. In part ii), it will be proven that these "reduced" matrices have only one nonzero entry in each row and column. Finally, in part iii), depending on the assumptions (13) and (22), it will be shown that the optimal $\boldsymbol{F}$ is a rectangular diagonal matrix.

Since the rank of the MIMO channel is $P$, in the sequel the dimension of $\boldsymbol{F}$ will be assumed to be $P \times n_{i}$. A more general case would consider $\boldsymbol{F}$ as an $n_{T} \times n_{i}$ matrix, but due to the rank of the channel, loading the last $n_{T}-P$ eigenmodes (with zero eigenvalues) would be a waste of transmit power. Therefore, without loss of generality, the last $n_{T}-P$ rows are set to zero. Hence, for notational simplicity $F$ is assumed to be of dimension $P \times n_{i}$ until the end of this proof. In fact, the waterfilling algorithm will set the powers of the excess antennas to zero since $\lambda_{k}=0$ for $k>P$, cf. (12) and (27).

Also, note that the case $n_{i}>P$ is excluded in the analysis, i.e., the number of input streams is greater than the number of available eigenmodes. This will clearly waste the transmit power and will increase the MSE by introducing additional terms. When CSI is available at the transmitter, this case can be avoided by loading the channel with a sufficient number of input streams. Hence, for clarity of the proof, this case is ignored here. A more detailed analysis for this case can be found in [39].

In order to prove Theorem 3, the following proposition is established.

Proposition 2: $\boldsymbol{F} \boldsymbol{K}_{d d} \boldsymbol{F}^{H}$ and $\boldsymbol{W}^{H} \boldsymbol{B}_{d}^{H} \boldsymbol{B}_{d} \boldsymbol{W}$ are diagonal.

Proof: Postmultiplying (35) by $\boldsymbol{K}_{d d} \boldsymbol{F}^{H}$, we obtain

$\boldsymbol{W}^{H} \boldsymbol{B}_{d}^{H} \boldsymbol{B}_{d} \boldsymbol{K}_{d d} \boldsymbol{F}^{H}=\boldsymbol{W}^{H} \boldsymbol{B}_{d}^{H} \boldsymbol{B}_{d} \boldsymbol{W} \boldsymbol{F} \boldsymbol{K}_{d d} \boldsymbol{F}^{H}+\gamma \boldsymbol{F} \boldsymbol{K}_{d d} \boldsymbol{F}^{H}$.

Premultiplying (34a) by $\boldsymbol{W}^{H} \boldsymbol{B}_{d}^{H} \boldsymbol{B}_{d}$, we obtain

$$
\begin{aligned}
\boldsymbol{W}^{H} \boldsymbol{B}_{d}^{H} \boldsymbol{B}_{d} \boldsymbol{K}_{d d} \boldsymbol{F}^{H}= & \boldsymbol{W}^{H} \boldsymbol{B}_{d}^{H} \boldsymbol{B}_{d} \boldsymbol{W} \boldsymbol{F} \boldsymbol{K}_{d d} \boldsymbol{F}^{H} \\
& +\boldsymbol{W}^{H} \boldsymbol{B}_{d}^{H} \boldsymbol{B}_{d} \boldsymbol{W} \boldsymbol{\Lambda}_{n n} .
\end{aligned}
$$

Subtraction of (44) from (43) yields

$$
\gamma \boldsymbol{F} \boldsymbol{K}_{d d} \boldsymbol{F}^{H}=\boldsymbol{W}^{H} B_{d}^{H} \boldsymbol{B}_{d} \boldsymbol{W} \boldsymbol{\Lambda}_{n n} .
$$

Assume that the diagonal matrix $\Lambda_{n n}$ is nondegenerate (i.e., $\boldsymbol{\Lambda}_{n n} \neq c \boldsymbol{I}$ ) which is almost sure due to the randomness of $\boldsymbol{H}$ in (12). Clearly, the matrices $\boldsymbol{W}^{H} \boldsymbol{B}_{d}^{H} \boldsymbol{B}_{d} \boldsymbol{W}$ and $\boldsymbol{F} \boldsymbol{K}_{d d} \boldsymbol{F}^{H}$ are
Hermitian symmetric and the right-hand side is also multiplied by the diagonal matrix $\boldsymbol{\Lambda}_{n n}$. In such a case, equality holds if and only if $\boldsymbol{W}^{H} \boldsymbol{B}_{d}^{H} \boldsymbol{B}_{d} \boldsymbol{W}$ and $\boldsymbol{F} \boldsymbol{K}_{d d} \boldsymbol{F}^{H}$ are "diagonal" (postmultiplying a Hermitian symmetric matrix by a nondegenerate diagonal matrix scales the columns which destroys the symmetry, hence makes the equality impossible unless the Hermitian matrix itself is diagonal). In the case of a degenerate $\Lambda_{n n}$, i.e., $\boldsymbol{\Lambda}_{n n}=c \boldsymbol{I}$, a small perturbation matrix $\Delta \boldsymbol{\Lambda}_{n n}$ can be added to $\boldsymbol{\Lambda}_{n n}$ as in [28] to obtain $\tilde{\Lambda}_{n n}=\Lambda_{n n}+\Delta \boldsymbol{\Lambda}_{n n}$ so as to have each diagonal element different. Use of $\tilde{\Lambda}_{n n}$ in (45) instead of $\boldsymbol{\Lambda}_{n n}$ requires the diagonality of $\boldsymbol{F} \boldsymbol{K}_{d d} \boldsymbol{F}^{H}$ from the above discussion. Without loss of generality taking the limit $\Delta \mathbf{\Lambda}_{n n} \rightarrow \mathbf{0}$ preserves the diagonality of $\boldsymbol{F} \boldsymbol{K}_{d d} \boldsymbol{F}^{H}$ and $\boldsymbol{W}^{H} \boldsymbol{B}_{d}^{H} \boldsymbol{B}_{d} \boldsymbol{W}$.

a) Part I: Recall that $\boldsymbol{F}$ is a $P \times n_{i}$ matrix, similarly, $\boldsymbol{W}$ and $\boldsymbol{G}$ are $n_{i} \times P$ matrices. Also, from (34b) notice that $\boldsymbol{W}$, hence $\boldsymbol{G}$, have the same structure as $\boldsymbol{F}^{H}$, i.e., have zeros at the same positions, since all other terms in that equation are diagonal. Also remember that $\Lambda_{n n}$ is a $P \times P$ full-rank diagonal matrix. Postmultiplying (34a) by $\boldsymbol{W}^{H}$ and premultiplying (35) by $\boldsymbol{F}^{H}$, one can obtain the following set of equations:

$$
\begin{aligned}
\boldsymbol{K}_{d d} \boldsymbol{F}^{H} \boldsymbol{W}^{H} & =\boldsymbol{W} \boldsymbol{F} \boldsymbol{K}_{d d} \boldsymbol{F}^{H} \boldsymbol{W}^{H}+\boldsymbol{W} \boldsymbol{\Lambda}_{n n} \boldsymbol{W}^{H} \\
\boldsymbol{F}^{H} \boldsymbol{W}^{H} \boldsymbol{B}_{d}^{H} \boldsymbol{B}_{d} & =\boldsymbol{F}^{H} \boldsymbol{W}^{H} \boldsymbol{B}_{d}^{H} \boldsymbol{B}_{d} \boldsymbol{W} \boldsymbol{F}+\gamma \boldsymbol{F}^{H} \boldsymbol{F} .
\end{aligned}
$$

Since $W F$ is diagonal as stated in Proposition 1, from (46a), $\boldsymbol{W} \boldsymbol{\Lambda}_{n n} \boldsymbol{W}^{H}$ must also be diagonal. Similarly, since $\boldsymbol{B}_{d}$ is diagonal as demonstrated in Appendix I, from (46b), $\boldsymbol{F}^{H} \boldsymbol{F}$ must also be diagonal. Also recall from Proposition 2 that $\boldsymbol{F} \boldsymbol{K}_{d d} \boldsymbol{F}^{H}$ is diagonal.

We define those diagonal matrices as

$$
\begin{gathered}
W \boldsymbol{\Lambda}_{n n} \boldsymbol{W}^{H} \triangleq D_{1} \\
\boldsymbol{F}^{H} \boldsymbol{F} \triangleq \boldsymbol{D}_{2} \\
\boldsymbol{F} \boldsymbol{K}_{d d} \boldsymbol{F}^{H} \triangleq D_{3}
\end{gathered}
$$

where $D_{1}$ and $D_{2}$ are nonnegative definite $n_{i} \times n_{i}$ full rank diagonal matrices (i.e., all $n_{i}$ eigenmodes are assumed to be active) and $\boldsymbol{D}_{3}$ is a nonnegative definite $P \times P$ diagonal matrix with rank $n_{i}$. Clearly, when $P=n_{i}, F$ and $\boldsymbol{W}$ are full rank square matrices.

When $P>n_{i}$, a closer examination of (49) reveals that the rows of $\boldsymbol{F}$ corresponding to zeros on the main diagonal of $\boldsymbol{D}_{3}$ must be zero. This can be proven as follows. First, note that $\boldsymbol{K}_{d d}$ is a full-rank diagonal matrix by definition, hence, $\boldsymbol{F} \boldsymbol{K}_{d d}^{1 / 2}$ has zeros exactly at the same the position as $\boldsymbol{F}$. Let $\left[D_{3}\right]_{k, k}$ be such a zero element, then from (49) $\left[\boldsymbol{F} \boldsymbol{K}_{d d}^{1 / 2}\right]_{k, 1: P}\left[\boldsymbol{F} \boldsymbol{K}_{d d}^{1 / 2}\right]_{k, 1: P}^{H}=0$, where " $k, 1: P$ " means all $P$ elements on the $k$ th row. However, this is the definition of a vector norm which is equal to zero if and only if $\left[\boldsymbol{F} \boldsymbol{K}_{d d}^{1 / 2}\right]_{k, 1: P}=\mathbf{0}$. Therefore, $\boldsymbol{F}$ is a rank $n_{i}$ matrix with $P-n_{i}$ rows consisting of all zeros. Similarly, $\boldsymbol{W}$ can be shown to have $P-n_{i}$ columns with all-zero entries.

Therefore, without loss of generality, in the sequel, the "squeezed" versions of $\boldsymbol{F}, \boldsymbol{W}$ and $\boldsymbol{\Lambda}_{n n}$ matrices which will be denoted by $\overline{\boldsymbol{F}}, \overline{\boldsymbol{W}}$ and $\overline{\boldsymbol{\Lambda}}_{n n}$ will be used. The all-zero rows are deleted from $\boldsymbol{F}$ and $\boldsymbol{W}^{H}$ and the corresponding rows and columns of $\Lambda_{n n}$ are also removed. Observe that $\overline{\boldsymbol{F}}$ and $\overline{\boldsymbol{W}}$ are full-rank "square" matrices with dimensions $n_{i} \times n_{i}$. Also, $\bar{\Lambda}_{n n}$ is still a full rank square diagonal matrix. 
b) Part II: Considering (47) and (48), we can write $\boldsymbol{W}$ and $F$ as follows:

$$
\begin{aligned}
\overline{\boldsymbol{W}} & =\boldsymbol{D}_{1}^{1 / 2} \boldsymbol{U}_{W} \mathcal{P}^{H} \boldsymbol{\Lambda}_{n n}^{-1 / 2} \\
\overline{\boldsymbol{F}} & =\mathcal{P} \boldsymbol{U}_{F} \boldsymbol{D}_{2}^{1 / 2}
\end{aligned}
$$

for some unitary matrices $U_{W}$ and $\boldsymbol{U}_{F}$ and a permutation matrix $\mathcal{P}$ (since the rows of $\boldsymbol{F}$ are our primary concern in the case $P>n_{i}$, the permutation matrix is set to permute the rows). Note that $\mathcal{P}^{H} \mathcal{P}=\mathcal{P} \mathcal{P}^{H}=\boldsymbol{I}$. The diagonal matrix $\overline{\boldsymbol{W}} \overline{\boldsymbol{F}}$ can be re-expressed in terms of these definitions as $\overline{\boldsymbol{W}} \overline{\boldsymbol{F}}=$ $\boldsymbol{D}_{1}^{1 / 2} \boldsymbol{U}_{W} \mathcal{P}^{H} \Lambda_{n n}^{-1 / 2} \mathcal{P} \boldsymbol{U}_{F} \boldsymbol{D}_{2}^{1 / 2}$, hence

$$
\boldsymbol{U}_{W} \mathcal{P}^{H} \overline{\boldsymbol{\Lambda}}_{n n}^{-1 / 2} \mathcal{P} \boldsymbol{U}_{F}=\boldsymbol{D}_{4}
$$

where $\boldsymbol{D}_{4}$ is a full rank $n_{i} \times n_{i}$ diagonal matrix. Note that $\mathcal{P}^{H} \overline{\boldsymbol{\Lambda}}_{n n}^{-1 / 2} \mathcal{P}$ is still diagonal. Now, $\boldsymbol{D}_{4}^{2}$ can be written as

$$
\begin{aligned}
& \boldsymbol{D}_{4} \boldsymbol{D}_{4}^{H}=\boldsymbol{U}_{W} \mathcal{P}^{H} \overline{\boldsymbol{\Lambda}}_{n n}^{-1} \mathcal{P} \boldsymbol{U}_{W}^{H} \\
& \boldsymbol{D}_{4}^{H} \boldsymbol{D}_{4}=\boldsymbol{U}_{F}^{H} \mathcal{P}^{H} \overline{\boldsymbol{\Lambda}}_{n n}^{-1} \mathcal{P} \boldsymbol{U}_{F}
\end{aligned}
$$

where both lines are equal. Hence, also considering (51), these equations imply that

$$
\boldsymbol{U}_{W}=\boldsymbol{U}_{F}=\operatorname{diag}\left\{e^{j \theta_{1}}, e^{j \theta_{2}}, \ldots, e^{j \theta_{n_{i}}}\right\}=\boldsymbol{\Theta}
$$

for arbitrary $\theta_{k} \in[0,2 \pi)$. Substitution of (50b) into (19) yields

$$
\begin{aligned}
J\left(\boldsymbol{F}, \boldsymbol{B}_{d}\right)= & \frac{1}{2 \pi} \operatorname{tr} \int_{-\pi}^{\pi} \boldsymbol{B}_{d}\left[\boldsymbol{K}_{d d}^{-1}\right. \\
& \left.+\boldsymbol{D}_{2}^{1 / 2} \boldsymbol{\Theta}^{H} \mathcal{P}^{H} \overline{\boldsymbol{\Lambda}}_{n n}^{-1} \mathcal{P} \boldsymbol{\Theta} \boldsymbol{D}_{2}^{1 / 2}\right]^{-1} \boldsymbol{B}_{d}^{H} d \omega \\
= & \frac{1}{2 \pi} \operatorname{tr} \int_{-\pi}^{\pi} \boldsymbol{B}_{d}\left[\boldsymbol{K}_{d d}^{-1}+\boldsymbol{D}_{2} \mathcal{P}^{H} \overline{\boldsymbol{\Lambda}}_{n n}^{-1} \mathcal{P}\right]^{-1} \boldsymbol{B}_{d}^{H} d \omega
\end{aligned}
$$

where the second equality follows from the fact that all matrices in the bracket are diagonal hence they commute. It can also be shown that the same property holds for the power constraint in (20b), meaning that the optimization is insensitive to the selection of $\boldsymbol{\Theta}$. Hence, without loss of optimality, we can set $\boldsymbol{\Theta}=\boldsymbol{I}$. Therefore, we can conclude from (50) that $\bar{W}$ and $\bar{F}$ are diagonal up to a permutation. This guarantees that there is at most one nonzero entry in the rows and columns of $\boldsymbol{F}$ and $\boldsymbol{W}$.

c) Part III: In this subsection, a sketch of the proof for the optimality of choosing the transmitter filter as diagonal is provided for the ordering (22).

Let the diagonal elements of $\boldsymbol{F}_{d}(\omega)$ be $f_{d, i}(\omega)$. It can be shown that when $P \geq n_{i}$, the MSE in (19) can be written for $\boldsymbol{F}_{d}(\omega)$ as

$$
J_{d}\left(\boldsymbol{F}_{d}, \boldsymbol{B}_{d}\right)=\frac{1}{2 \pi} \int_{-\pi}^{\pi} \sum_{i=1}^{n_{i}} \frac{\kappa_{i}\left|b_{d, i}\right|^{2} \lambda_{i}}{\left|f_{d, i}\right|^{2} \kappa_{i}+\lambda_{i}} d \omega
$$

where $\boldsymbol{B}_{d}(\omega)$ is diagonal with components $b_{d, i}(\omega)$ as proven before. Define the permuted matrix $\boldsymbol{F}_{p}(\omega)$ which is identical to $\boldsymbol{F}_{d}(\omega)$ everywhere except the $j$ th and $k$ th $(j<k)$ rows of $\boldsymbol{F}_{d}(\omega)$ which are permuted only at frequency $\omega=\omega_{o}$

$$
\boldsymbol{F}_{p}(\omega)=\mathcal{P} \boldsymbol{F}_{d}(\omega) .
$$

It can easily be shown that this permutation does not affect $\boldsymbol{K}_{d d}$ or $\boldsymbol{B}_{d}$ but swaps the $j$ th and $k$ th entries of $\boldsymbol{\Lambda}_{n n}^{-1}$, i.e., $\boldsymbol{\Lambda}_{n n, p}^{-1}=$ $\mathcal{P}^{H} \Lambda_{n n}^{-1} \mathcal{P}$ is still a diagonal matrix with diagonal entries $\lambda_{p, i}^{-1}$. Therefore, the MSE for the permutation can be written as

$$
J_{p}\left(\boldsymbol{F}_{p}, \boldsymbol{B}_{d}\right)=\frac{1}{2 \pi} \int_{-\pi}^{\pi} \sum_{i=1}^{n_{i}} \frac{\kappa_{i}\left|b_{d, i}\right|^{2} \lambda_{p, i}}{\left|f_{p, i}\right|^{2} \kappa_{i}+\lambda_{p, i}} d \omega .
$$

The difference between $J_{d}\left(\boldsymbol{F}_{d}, \boldsymbol{B}_{d}\right)$ and $J_{p}\left(\boldsymbol{F}_{p}, \boldsymbol{B}_{d}\right)$ is defined as

$$
\begin{aligned}
\Delta J & =J_{p}\left(\boldsymbol{F}_{p}(\omega), \boldsymbol{B}_{d}(\omega)\right)-J_{d}\left(\boldsymbol{F}_{d}(\omega), \boldsymbol{B}_{d}(\omega)\right) \\
& = \begin{cases}\left(\frac{\kappa_{j}\left|b_{d, j}\right|^{2} \lambda_{p, j}}{\left|f_{p, j}\right|^{2} \kappa_{j}+\lambda_{p, j}}+\frac{\kappa_{k}\left|b_{d, k}\right|^{2} \lambda_{p, k}}{\left|f_{p, k}\right|^{2} \kappa_{k}+\lambda_{p, k}}\right), & \omega=\omega_{o} \\
-\left(\frac{\kappa_{j}\left|b_{d, j}\right|^{2} \lambda_{j}}{\left|f_{d, j}\right|^{2} \kappa_{j}+\lambda_{j}}+\frac{\kappa_{k}\left|b_{d, k}\right|^{2} \lambda_{k}}{\left|f_{d, k}\right|^{2} \kappa_{k}+\lambda_{k}}\right) & \text { otherwise } \\
0, & \end{cases}
\end{aligned}
$$

since the permutation is effective only at $\omega=\omega_{o}$ and for the pair $j$ and $k$, and both scenarios are identical elsewhere. In order to satisfy the power constraint, the following equality must hold:

$$
\left|f_{p, j}\right|^{2} \kappa_{j}+\left|f_{p, k}\right|^{2} \kappa_{k}=\left|f_{d, j}\right|^{2} \kappa_{j}+\left|f_{d, k}\right|^{2} \kappa_{k} .
$$

First, consider the case

$$
\frac{\left|f_{p, j}\right|^{2} \kappa_{j}}{\lambda_{p, j}} \leq \frac{\left|f_{p, k}\right|^{2} \kappa_{k}}{\lambda_{p, k}}
$$

Then select $f_{d, j}$ and $f_{d, k}$ such that

$$
\left|f_{d, j}\right|^{2} \kappa_{j}=\left|f_{p, k}\right|^{2} \kappa_{k} \quad \text { and } \quad\left|f_{d, k}\right|^{2} \kappa_{k}=\left|f_{p, j}\right|^{2} \kappa_{j}
$$

which clearly satisfies (55). Substitution of (57) into (54) yields

$$
\begin{aligned}
\Delta J=\left(\kappa_{j}\left|b_{d, j}\right|^{2}-\right. & \left.\kappa_{k}\left|b_{d, k}\right|^{2}\right) \\
& \times\left(\frac{\lambda_{p, j}}{\left|f_{p, j}\right|^{2} \kappa_{j}+\lambda_{p, j}}-\frac{\lambda_{p, k}}{\left|f_{p, k}\right|^{2} \kappa_{k}+\lambda_{p, k}}\right)
\end{aligned}
$$

which is nonnegative because $\kappa_{j}\left|b_{d, j}\right|^{2} \geq \kappa_{k}\left|b_{d, k}\right|^{2}$ from assumption (22). The second parenthesis follows from the assumption (56).

For the second case, i.e., if

$$
\frac{\left|f_{p, j}\right|^{2} \kappa_{j}}{\lambda_{p, j}}>\frac{\left|f_{p, k}\right|^{2} \kappa_{k}}{\lambda_{p, k}}
$$

we set $f_{d, j}$ and $f_{d, k}$ as

$$
\left|f_{d, j}\right|^{2} \kappa_{j}=\frac{\lambda_{p, k}}{\lambda_{p, j}}\left|f_{p, j}\right|^{2} \kappa_{j}
$$

and

$$
\left|f_{d, k}\right|^{2} \kappa_{k}=\frac{\lambda_{p, j}}{\lambda_{p, k}}\left|f_{p, k}\right|^{2} \kappa_{k} .
$$

Substituting (59) into (54), we obtain

$$
\begin{aligned}
\Delta J= & \kappa_{j}\left|b_{d, j}\right|^{2}\left(\frac{\lambda_{p, j}}{\left|f_{p, j}\right|^{2} \kappa_{j}+\lambda_{p, j}}-\frac{\lambda_{p, j}}{\left|f_{p, j}\right|^{2} \kappa_{j}+\lambda_{p, j}}\right) \\
& +\kappa_{k}\left|b_{d, k}\right|^{2}\left(\frac{\lambda_{p, k}}{\left|f_{p, k}\right|^{2} \kappa_{k}+\lambda_{p, k}}-\frac{\lambda_{p, k}}{\left|f_{p, k}\right|^{2} \kappa_{k}+\lambda_{p, k}}\right) \\
= & 0
\end{aligned}
$$


which means both scenarios end up with the same MSE. However, looking from the transmitted power point of view

$$
\begin{aligned}
\frac{\left|f_{p, j}\right|^{2} \kappa_{j}+\left|f_{p, k}\right|^{2} \kappa_{k}}{\left|f_{d, j}\right|^{2} \kappa_{j}+\left|f_{d, k}\right|^{2} \kappa_{k}} & =\frac{\left|f_{p, j}\right|^{2} \kappa_{j}+\left|f_{p, k}\right|^{2} \kappa_{k}}{\frac{\lambda_{p, k}\left|f_{p, j}\right|^{2} \kappa_{j}+\frac{\lambda_{p, j}}{\lambda_{p, k}}\left|f_{p, k}\right|^{2} \kappa_{k}}{\lambda_{p, j}}} \\
& =\frac{\lambda_{p, j} \frac{\left|f_{p, j}\right|^{2} \kappa_{j}}{\lambda_{p, j}}+\lambda_{p, k} \frac{\left|f_{p, k}\right|^{2} \kappa_{k}}{\lambda_{p, k}}}{\lambda_{p, k} \frac{\left|f_{p, j}\right|^{2} \kappa_{j}}{\lambda_{p, j}}+\lambda_{p, j} \frac{\left|f_{p, k}\right|^{2} \kappa_{k}}{\lambda_{p, k}}} \geq 1
\end{aligned}
$$

which can be shown to be greater than or equal to one under the assumptions (13) and (58). Note that $\lambda_{p, j}=\lambda_{k}$ and $\lambda_{p, k}=$ $\lambda_{j}$. The relation in (60) and (61) means that the same MSE is achieved while the permuted scenario needs more transmit power. Since the MSE is monotonically related to the transmit power, the diagonal scenario yields a lower MSE than the permuted scenario when the transmit power of both scenarios are set equal.

The argument in this section is valid for each frequency $\omega$ and all pairs $j$ and $k$. Hence, repeating the algorithm for all of the frequencies and pairs confirms that the diagonal scenario yields the lowest MSE among all permutation scenarios. Hence, the optimum permutation is $\mathcal{P}(\omega)=\boldsymbol{I}$. With this permutation, (53) yields (24).

This concludes the proof of the diagonality of the optimum transmitter filter for the ordering (22).

\section{REFERENCES}

[1] C. Toker, S. Lambotharan, and J. A. Chambers, "Joint transmitter and receiver design for MIMO channel shortening," in Proc. IEEE Int. Conf. Acoust., Speech, Signal Processing, Mar. 2005, vol. 4, pp. 909-912.

[2] IEEE Std. 802.11 [Online]. Available: http://grouper.ieee.org/groups/ 802/11/, correct as of Oct. 2006

[3] ETSI HIPERLAN2 Strd. [Online]. Available: http://portal.etsi.org/ radio/HiperLAN/HiperLAN.asp, correct as of Oct. 2006

[4] ETSI DAB Std. [Online]. Available: http://portal.etsi.org/radio/Digita1AudioBroadcasting/DAB.asp, correct as of Oct. 2006

[5] ETSI DVB Std. [Online]. Available: http://www.dvb.org, correct as of Oct. 2006

[6] A. J. Paulraj and C. B. Papadias, "Space-time processing for wireless communications," IEEE Signal Process. Mag., vol. 6, pp. 49-83, Nov. 1997.

[7] E. G. Larsson et al., "Space-time block coding for frequency-selective channels," in Proc. IEEE Int. Conf. Acoust., Speech, Signal Process, May 2002, vol. 3, pp. 13-17.

[8] E. Lindskog and A. Paulraj, "A transmit diversity scheme for channels with intersymbol interference," in Proc. IEEE Int. Conf. Commun., Jun. 2000, vol. 1, pp. 307-311.

[9] E. G. Larsson and P. Stoica, Space-Time Block Coding for Wireless Communications. Cambridge, U.K.: Cambridge Univ. Press, 2003.

[10] V. Tarokh, A. Naguib, N. Seshadri, and A. R. Calderbank, "Space-time codes for high data rate wireless communication: Performance analysis and code construction," IEEE Trans. Inf. Theory, vol. 44, no. 2, pp. 744-765, Mar. 1998.

[11] B. Vucetic and J. Yuan, Space-Time Coding. West Sussex, U.K.: Wiley, 2003.

[12] J. F. Hayes, "The Viterbi algorithm applied to digital data transmission," IEEE Commun. Mag., vol. 13, no. 2, pp. 15-20, Mar. 1975.

[13] D. D. Falconer and F. R. Magee, "Adaptive channel memory truncation for maximum likelihood sequence estimation," Bell Syst. Tech. J., pp. 1541-1562, Nov. 1973.

[14] N. Al-Dhahir and J. M. Cioffi, "Optimum finite-length equalization for multicarrier transceivers," IEEE Trans. Commun., vol. 44, no. 1, pp. 56-64, Jan. 1996.

[15] R. K. Martin et al., "Unification and evaluation if equalization structures and design algorithms for discrete multitone modulation systems," IEEE Trans. Signal Process., vol. 53, no. 10, pp. 3880-3894, Oct. 2005.
[16] G. Arslan, "Equalization of Discrete Multitone Transceivers," Ph.D dissertation, Univ. Texas, Austin, Dec. 2000 [Online]. Available: http:// www.ece.utexas.edu/ bevans/students/phd/guner_arslan/phd.pdf, correct as of Oct. 2006

[17] M. Milošević, "Maximizing Data Rate of Discrete Multitone Systems Using Time Domain Equalization Design,” Ph.D. dissertation, Univ. Texas, Austin, May 2003 [Online]. Available: http://www.ece.utexas. edu/ bevans/students/phd/milos_milosevic/phd.pdf, correct as of Oc. 2006

[18] J. S. Chow and J. M. Cioffi, "A cost-effective maximum likelihood receiver for multicarrier systems," in Proc. IEEE Int. Conf. Commun., Jun. 1992, vol. 2, pp. 948-952.

[19] N. Al-Dhahir and J. M. Cioffi, "Efficiently computed reduced-parameter input-aided MMSE equalizers for ML detection: A unified approach," IEEE Trans. Inf. Theory, vol. 42, no. 3, pp. 903-915, May 1996.

[20] N. Al-Dhahir, A. F. Naguib, and A. R. Calderbank, "Finite-length MIMO decision feedback equalization for space-time block-coded signals over multipath-fading channels," IEEE Trans. Veh. Technol., vol. 50, no. 4, pp. 1176-1182, Jul. 2001.

[21] P. J. W. Melsa, R. C. Younce, and C. E. Rohrs, "Impulse response shortening for discrete multitone transceivers," IEEE Trans. Commun., vol. 42, no. 12, pp. 1662-1672, Dec. 1996.

[22] G. Arslan, B. L. Evans, and S. Kiaei, "Optimum channel shortening for discrete multitone transceivers," in Proc. IEEE Int. Conf. Acoust., Speech, Signal Process, Jun. 2000, vol. 5, pp. 2965-2968.

[23] _ "Equalization for discrete multitone receivers to maximize bit rate," IEEE Trans. Signal Process., vol. 49, no. 12, pp. 3123-3135, Dec. 2001.

[24] K. Van Acker et al., "Per tone equalization for DMT-based systems," IEEE Trans. Commun., vol. 49, no. 1, pp. 109-119, Jan. 2001.

[25] N. Al-Dhahir, "FIR channel shortening equalizers for MIMO ISI channels," IEEE Trans. Commun., vol. 49, no. 2, pp. 213-218, Feb. 2001.

[26] C. Toker et al., "Joint spatial and temporal channel shortening techniques for frequency selective fading MIMO channels," Proc. Inst. Elec. Eng.-Commun., vol. 152, no. 1, pp. 89-94, Feb. 2005.

[27] A. Scaglione et al., "Optimal designs for space-time linear precoders and decoders," IEEE Trans. Signal Process., vol. 50, no. 5, pp. 1051-1064, May 2002

[28] H. Sampath, P. Stoica, and A. Paulraj, "Generalized linear precoder and decoder design for MIMO channels using the weighted MMSE criterion," IEEE Trans. Commun., vol. 49, no. 12, pp. 2198-2206, Dec. 2001.

[29] T. Berger and D. W. Tufts, "Optimum pulse amplitude modulation Part I: Transmitter-receiver design and bounds from information theory," IEEE Trans. Inf. Theory, vol. IT-13, no. 2, pp. 196-208, Apr. 1967.

[30] J. Yang and S. Roy, "Joint transmitter-receiver optimization for multiinput multi-output systems with decision feedback," IEEE Trans. Inf. Theory, vol. 40, no. 5, pp. 1334-1347, Sep. 1994.

[31] — - "On joint transmitter and receiver optimization for multiple-input-multiple-output (MIMO) transmission systems," IEEE Trans. Commun., vol. 42, no. 12, pp. 3221-3231, Dec. 1994.

[32] R. K. Martin et al., "Infinite length results and design implications for time-domain equalizers," IEEE Trans. Signal Process., vol. 52, no. 1, pp. 297-301, Jan. 2004.

[33] J. M. Cioffi, EE 379C Advanced Digital Communications, Stanford Univ., Stanford, CA, 1998 [Online]. Available: http://www.stanford. edu/class/ee379c, correct as of Oct. 2006

[34] A. Scaglione, S. Barbarossa, and G. B. Giannakis, "Filterbank transceivers optimizing information rate in block transmissions over dispersive channels," IEEE Trans. Inf. Theory, vol. 45, no. 3, pp. 1019-1032, Apr. 1999.

[35] D. P. Palomar and M. A. Lagunas, "Joint transmit-receive space-time equalization in spatially correlated MIMO channels: A beamforming approach," IEEE J. Sel. Areas Commun., vol. 21, no. 5, pp. 730-743, Jun. 2003.

[36] E. Kreyszig, Advanced Engineering Mathematics, 8th ed. New York: Wiley, 1999.

[37] C. Toker et al., "Channel shortening filter design based on polynomial methods," in Proc. IEEE Veh. Technol. Conf.-Spring, May 2004, pp. $565-569$.

[38] C. Toker, S. Lambotharan, and J. A. Chambers, "Infinite length channel shortening filtering based on polynomial approach," in Proc. Europ. Signal Processing Conf. (EUSIPCO'04), Vienna, Austria, Sep. 2004, pp. 2223-2226. 
[39] C. Toker, "Signal Processing Algorithms and Architectures for Communication Transceivers," Ph.D. dissertation, King's College London, Univ. London, London, U.K., Oct. 2004 [Online]. Available: http:// www.ee.hacettepe.edu.tr/ toker/Toker_PhD_Dissertation.pdf, correct as of Oct. 2006

[40] S. Boyd and L. Vanderberghe, Convex Optimization. Cambridge, U.K.: Cambridge Univ. Press, 2004.

[41] D. P. Palomar et al., "Practical implementation of jointly designed transmit receive space-time IIR filters," in Proc. IEEE 6th Int. Symp. Signal Processing and Its Applications, Aug. 2001, vol. 2, pp. 521-524.

[42] N. Al-Dhahir and J. M. Cioffi, "MMSE decision-feedback equalizers: Finite-length results," IEEE Trans. Inf. Theory, vol. 41, no. 4, pp. 961-975, Jul. 1995.

[43] R. A. Casas, P. B. Schniter, J. Balakrishnan, and C. R. Johnson Jr., DFE Tutorial Jul. 1998 [Online]. Available: http://www.ece.osu.edu/ $\sim$ schniter/postscript/dfetutorial.pdf, Cornell Uinv. BERG, correct as of Oct. 2006

[44] G. H. Golub and C. F. Van Loan, Matrix Computations, 3rd ed. Baltimore, MD: Johns Hopkins Univ. Press, 1996.

[45] Z. Shen, J. G. Andrews, R. W. Heath, Jr., and B. L. Evans, "Low complexity user selection algorithms for multiuser MIMO systems with block diagonalization," IEEE Trans. Signal Process., vol. 54, no. 9, pp. 3658-3663, Sep. 2006.

[46] Texas Instruments TMS320C67x Instruction Set, [Online]. Available: http://focus.ti.com/lit/ug/spru733/spru733.pdf, correct as of Oct. 2006

[47] N. Al-Dhahir, A. H. Sayed, and J. M. Cioffi, "Stable pole-zero modeling for long FIR filters with applications to the MMSE-DFE," IEEE Trans. Commun., vol. 45, no. 5, pp. 508-513, May 1997.

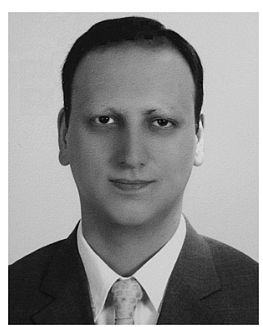

Cenk Toker (S'97-M'05) was born in Ankara, Turkey, in 1974. He received the B.Sc. degree inelectrical and electronics engineering with high honors from Hacettepe University, Ankara, Turkey, in 1995 and the M.Sc. degree in also in electrical and electronic engineering from the Middle East Technical University, Ankara, Turkey in 1999. He was awarded the Ph.D. degree in electrical engineering by the Centre for Digital Signal Processing (CDSPR), King's College London (KCL), U.K. in 2004.

Between 1995 and 2002, he held several positions in the industry as an R\&D engineer, including Motorola, Swindon, U.K., TUBITAK-BILTEN, Ankara, Turkey, and Aselsan Inc., Ankara, Turkey. In 2005, he continued his studies at CDSPR, KCL as a Postdoctoral Research Associate. Later, he joined the faculty of the Department of Electrical and Electronics Engineering, Hacettepe University in September, 2005, where he is currently an Assistant Professor. His research interests include joint transceiver design for multicarrier applications, multiple-input multiple-output (MIMO) signal processing, channel equalization-specifically channel shortening, space-time coding, resource allocation problems, and genetic algorithms.

In 2001, Dr. Toker received the Motorola Sponsorship for his doctoral studies through the University Partnership for Research Programme. From 2002 to 2004, he was sponsored by the School of Physical Sciences and Engineering of King's College London.

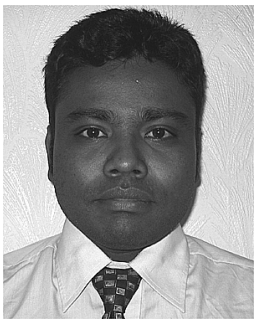

Sangarapillai Lambotharan (M'95-SM'06) received a first class B.Eng. honours degree in electrical and electronic engineering from City University, London, U.K. in 1994 and the Ph.D. degree in digital signal processing from Imperial College, London, U.K. in 1997, where he remained until 1999 as a Postdoctoral Research Associate.

He joined the Centre of Digital Signal Processing with the Cardiff School of Engineering in the University of Cardiff, Cardiff, Wales, U.K., as a Senior Lecturer in August 2005. He was a Visiting Scientist at the Engineering and Theory Centre of Cornell University, Ithaca, NY, in 1996. Between November 1999 and January 2002, he was with the Motorola Applied Research Group in Swindon before joining the Centre for DSP research at King's College London, U.K. as a Lecturer. His current research interests include equalization and interference cancellation techniques for multiple-input multiple-output (MIMO) systems, channel shortening filters for multitone communication systems, and statistical parameter estimation and detection techniques. He has published around 70 technical journal and conference papers in signal processing for communications. He is an Associate Editor for the EURASIP Journal on Wireless Communications and Networking.

Dr. Lambotharan is a member of IEE.

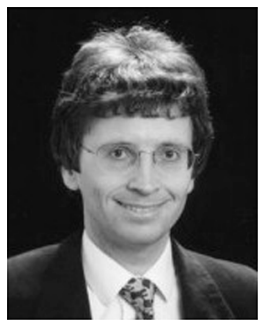

Jonathon A. Chambers (SM'98) was born in Peterborough, U.K., in 1960. He received the B.Sc. (Hons.) degree from the Polytechnic of Central London, U.K., in 1985, together with the Robert Mitchell Medal as the top graduate of the Polytechnic, and the Ph.D. degree from University of London, U.K., in 1990 after study at Peterhouse, Cambridge University, Cambridge, U.K., and Imperial College London.

He served in the Royal Navy as an Artificer Apprentice in Action, Data and Control between 1979 and 1982. He has since held academic and industrial positions with Bath University, Imperial College London, King's College London, and Schlumberger Cambridge Research. In January 2004, he became a Cardiff Professorial Fellow in Digital Signal Processing within the Cardiff School of Engineering, Cardiff, Wales, U.K. He leads a team of researchers involved in the analysis, design, and evaluation of novel algorithms for digital signal processing with application in acoustics, biomedicine, and wireless communications. His research contributions have been in adaptive and blind signal processing. He has authored and/or coauthored approximately 250 conference and journal publications, and supervised $25 \mathrm{Ph}$.D. degree graduates.

Dr. Chambers is currently serving for a second time as an Associate Editor for the IEEE TRANSACTIONS ON Signal PROCESSING. He is a Member of the IEEE Technical Committee on Signal Processing Theory and Methods. He is a Past Chairman of the U.K. IEE Professional Group E5, Signal Processing, and an IEE Fellow. 